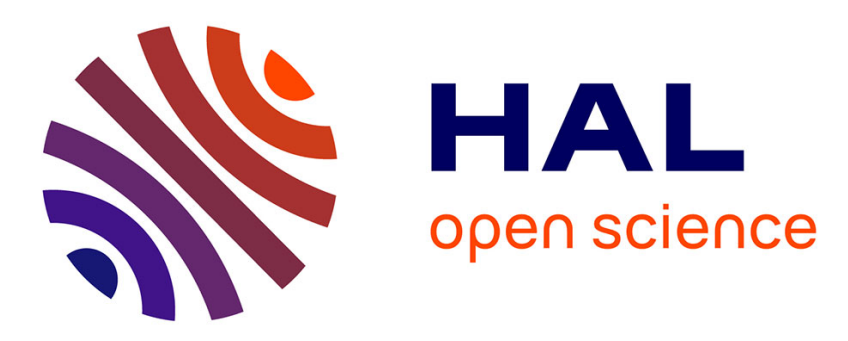

\title{
A numerical tool for the frequency domain simulation of large arrays of floating bodies in waves
}

Francesc H Fabregas-Flavia, Cameron Mcnatt, François Rongère, Aurélien Babarit, Alain H. Clément

\section{- To cite this version:}

Francesc H Fabregas-Flavia, Cameron Mcnatt, François Rongère, Aurélien Babarit, Alain H. Clément. A numerical tool for the frequency domain simulation of large arrays of floating bodies in waves. Ocean Engineering, 2018, 145, pp.299-311. 10.1016/j.oceaneng.2017.11.026 . hal-01635249

\section{HAL Id: hal-01635249 https://hal.science/hal-01635249}

Submitted on 15 Mar 2019

HAL is a multi-disciplinary open access archive for the deposit and dissemination of scientific research documents, whether they are published or not. The documents may come from teaching and research institutions in France or abroad, or from public or private research centers.
L'archive ouverte pluridisciplinaire HAL, est destinée au dépôt et à la diffusion de documents scientifiques de niveau recherche, publiés ou non, émanant des établissements d'enseignement et de recherche français ou étrangers, des laboratoires publics ou privés. 


\title{
A numerical tool for the frequency domain simulation of large arrays of identical floating bodies in waves
}

\author{
F. Fàbregas Flaviàa, , C. McNatt ${ }^{\mathrm{b}}$, F. Rongère ${ }^{\mathrm{a}}$, A. Babarit ${ }^{\mathrm{a}}$, A. H. Clément $^{\mathrm{a}}$ \\ ${ }^{a}$ Ecole Centrale de Nantes, LHEEA, UMR CNRS 6598, Nantes (France) \\ ${ }^{b}$ The University of Edinburgh, Institute for Energy Systems, Edinburgh (UK)
}

\begin{abstract}
The finite-depth interaction theory (IT) introduced by Kagemoto H. and Yue (1986) enables one to drastically speed up the computation of the added mass, damping and excitation force coefficients of a group ("farm") of floating bodies when compared to direct calculations with standard widely available boundary element method (BEM) codes. An essential part of the theory is the calculation of two hydrodynamic operators, which characterize the way a body diffracts and radiates waves, known as Diffraction Transfer Matrix (DTM) and Radiation Characteristics (RC) respectively. Two different strategies to compute them for arbitrary geometries have been proposed in the literature (Goo, J.-S. and Yoshida, 1990 McNatt J. C. et al. 2015). The purpose of this study is to present the implementation of the former in the zeroth-order BEM solver $\mathrm{NEMOH}$ and to compare it with the latter by providing an insight into the DTM and the RC of a truncated vertical circular cylinder and a square box. A very good agreement between the hydrodynamic operators computed with both methodologies is obtained. In addition, hydrodynamic coefficients generated by means of the IT are verified against direct NEMOH calculations for two different array layouts. Results show the effect of hydrodynamic interactions as well as the importance of the evanescent modes truncation for closely spaced configurations.
\end{abstract}

\footnotetext{
${ }^{*}$ Corresponding author

Email address: francesc.fabregas-flavia@ec-nantes.fr (F. Fàbregas Flavià)
} 
Keywords: BEM, Interaction Theory, Diffraction Transfer Matrix, Radiation Characteristics, Wave Farms

\section{Introduction}

Because of the limits of the energy conversion capacity of single devices, it is nowadays well-accepted that commercial exploitation of wave energy will require the deployment of wave energy converters (WECs) in array. As the advancement of WEC technology continues, there is an increasing interest in developing numerical tools to investigate how WECs will interact with one another in the first generation farms.

It has been shown that wave interactions may affect the forces acting upon the WECs and the energy production of the wave farm to varying degrees de10 pending on the layout (Budal, 1977, Falnes J. and Budal, 1982, Falnes, 1984). Forces due to wave radiation and scattering in the array can be well represented by matrices of linear radiation and excitation force coefficients. However, due to memory and time restrictions, the direct computation of these matrices for large arrays of bodies $O(100)$ is beyond the capabilities of standard Boundary Element Method (BEM) codes.

The methodology developed by Kagemoto H. and Yue (1986), known as Direct Matrix Method interaction theory and that we shall refer to herein as IT, combines the features of the Direct Matrix approach in Spring B. H. and Monkmeyer P. (1974) and Simon (1982), and the multiple-scattering technique by Twersky (1952) and Ohkusu (1974). It enables one to accelerate the computation of the hydrodynamic coefficients, for multi-body arrays under certain circumstances, including finite water depth and no vertical overlap. IT computations can generate the coefficients for large arrays, which could not be computed directly with a BEM code. The IT computation is based on mathematically characterizing how an individual isolated device scatters and radiates waves. For this, two hydrodynamic operators known as Diffraction Transfer Matrix and Radiation Characteristics that we shall refer to herein as DTM and 
RC respectively need to be computed. Kagemoto H. and Yue (1986) provided at method to obtain the DTM and RC for axisymmetric bodies. Goo, J.-S. and 30 Yoshida (1990) developed an approach based on a cylindrical representation of the Green's function by Fenton (1978) to calculate the elements of the DTM and $\mathrm{RC}$ for an arbitrary geometry using a BEM.

The approach by Goo, J.-S. and Yoshida (1990) was used to study the forces on the fixed (Chakrabarti, 2000) and floating (Chakrabarti, 2001) modules of 35 an interconnected multi-moduled floating offshore structure used by the US Navy. It was also employed by Peter M. A. and Meylan H. (2004) to study the interactions between ocean waves and large fields of ice floes in the marginal ice zone. For that, the extension of the theory to infinite-depth was required. Based on Kagemoto H. and Yue (1986), Kashiwagi (2000) derived a hierarchical 40 interaction theory aimed at studying hydrodynamic interactions among a great number of bodies in very large floating structures. More recently, in the context of wave attenuation in the marginal ice zone, Montiel F. et al. (2016) proposed an approach known as slab-clustering method which combines the Direct Matrix Method with a one dimension multiple scattering technique to solve the multiple-scattering problem in arrays composed of thousands of ice floes.

The methodology of Goo, J.-S. and Yoshida (1990) requires the modification of the standard diffraction problem boundary conditions, as well as access to the source strength distribution on the discretized wetted surface of the body. This output is not accessible to the user in the majority of standard BEM codes which only provide the standard hydrodynamic excitation forces and radiation coefficients after integration over the body surface. As a result, the IT has been applied mainly in cases where WEC geometries are such that an analytical expression of its hydrodynamic operators exists (Child B. and Venugopal, 2010. Göteman, M. et al. 2015). To overcome such limitation, McNatt J. C. et al. 55 2015) developed and validated an alternative approach to the one of Goo, J.-S. and Yoshida (1990) to calculate the DTM and RC using the standard output of 
available BEM codes like WAMIT 1 A shortcoming of the method provided by McNatt J. C. et al. (2015) is that it is unable to include evanescent wave modes in the IT computation.

60 A key goal of this study is to verify the outputs of a new implementation of the method developed by Goo, J.-S. and Yoshida (1990) in the open-source, ${ }_{\mathrm{B}} \mathrm{BEM}$-code NEMOH${ }^{2}$ to the outputs using the method developed by McNatt J. C. et al. (2015) by comparing the DTM and the RC of two different geometries, a truncated vertical circular cylinder and a cube. This comparison also serves to illustrate the frequency-dependent patterns of the DTM and RC, which, despite their necessity in IT, have not received much attention in literature.

In the following sections, we first present the solution to the Boundary Value Problem (BVP) for an isolated body in cylindrical coordinates and introduce the concept of partial cylindrical waves. We then consider the multi-body BVP and its exact algebraic solution by means of the IT method derived by Kagemoto H. and Yue (1986). The procedure to obtain the radiation and excitation force coefficients from the solution to the multiple-scattering problem is also presented. Following that, the methodologies of Goo, J.-S. and Yoshida (1990) and McNatt J. C. et al. (2015) for computing the DTM and the RC are presented and com75 pared in section 3. Details of the numerical implementation of the procedure by Goo, J.-S. and Yoshida (1990) in the open-source BEM solver NEMOH are given in section 4 . Section 5 presents numerical results as the hydrodynamic operators for a truncated vertical cylinder and a cube. Verifications of IT with direct BEM computations are made via comparison of the free surface elevation and the hydrodynamic coefficients. These results show the importance of selecting the correct truncation value for cases where bodies are placed in close proximity, which has not been shown in previous studies. Finally, verification of the hydrodynamic coefficients computed by NEMOH is made by comparison of a semi-analytical solution for vertical cylinders in a particular array layout

\footnotetext{
${ }^{1}$ www.wamit.com

${ }^{2}$ http://lheea.ec-nantes.fr/doku.php/emo/nemoh/start
} 
which includes near trapped-modes.

\section{Interaction Theory}

п The Direct Matrix Method interaction theory (IT) by Kagemoto H. and Yue (1986) is based on the linear potential flow theory (Newman, J.N, 1999). Thus, the constraints of linearity of the governing equations and perfect fluid characteristics are assumed to be satisfied. The former applies as long as a small wave steepness and a small amplitude of the body motions with respect to its characteristic dimension can be assumed. The latter holds if the fluid can be characterized as inviscid and incompressible and the flow as irrotational. In this case all the flow quantities of interest can be derived from a scalar field known as velocity potential $\Phi$ and such that $\vec{v}=\nabla \Phi$. If in addition, a harmonic time dependence is assumed, the spatial and time variation of $\Phi$ can be decoupled as $\Phi=\operatorname{Re}\left\{\phi(x, y, z) e^{-i \omega t}\right\}$, where $\phi$ is the complex spatial part of $\Phi,(x, y, z)$ are the spatial coordinates in a global Cartesian reference system, $i=\sqrt{-1}, \omega$ the angular frequency and $t$ the time.

For an array of floating bodies, and given the linearity of the problem, the total potential in the fluid domain can be computed as a superposition of the different forms of the velocity potential:

$$
\phi=\phi^{I}+\sum_{j=1}^{N_{b}} \phi_{j}^{S}+\sum_{j=1}^{N_{b}} \sum_{k=1}^{D f_{j}} \phi_{j}^{R, k}
$$

where $\phi^{I}$ is the ambient incident wave potential, $\phi_{j}^{S}$ is the scattered potential by body $j$ in the array when held fixed, $\phi_{j}^{R, k}$ is the radiated potential by body $j$ moving in its $k$ th degree of freedom, $N_{b}$ represents the number of bodies in the array and $D f_{j}$ stands for the number of degrees of freedom $k$ of body $j$.

\subsection{Partial Waves}

In a large array, waves emanating from each body due to scattering and radiation will propagate and interact with its neighbours. This will lead to a succession of scattering events which are referred to as multiple-scattering 


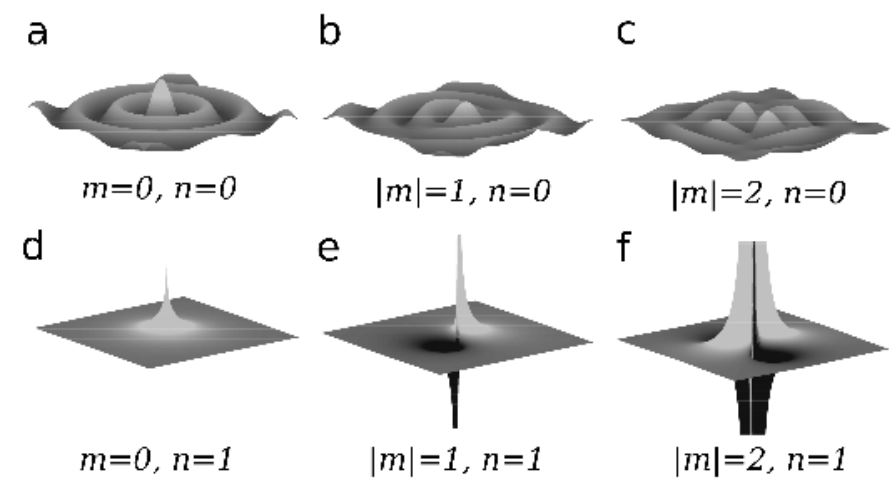

Figure 1: Partial waves modes. Progressive term $H_{m}^{(1)}(r)(\mathrm{a}, \mathrm{b}, \mathrm{c})$; evanescent term $K_{m}(r)$ $(d, e, f)$.

Martin, 2006). In this context, the representation of the scattered potential by body $j$ can be described by the outgoing wave solution to the BVP in cylindrical coordinates (a full derivation can be found in Child (2011) 3.4.1):

$$
\begin{aligned}
\phi_{j}^{S}=\sum_{m=-\infty}^{\infty}\left[\left(A_{j}^{S}\right)_{0 m} \frac{\cosh k_{0}\left(z_{j}+d\right)}{\cosh k_{0} d} H_{m}^{(1)}\left(k_{0} r_{j}\right)\right. \\
\left.+\sum_{n=1}^{\infty}\left(A_{j}^{S}\right)_{n m} \cos k_{n}\left(z_{j}+d\right) K_{m}\left(k_{n} r_{j}\right)\right] e^{i m \theta_{j}}
\end{aligned}
$$

where $H_{m}^{(1)}$ is the Hankel function of the first kind of order $m$ (see Figures 1 1 $1 p$ 1 1 ) , $K_{m}$ is the modified Bessel function of the second kind of order $m$ (see Figures $17,1,1 f),\left(A_{j}^{S}\right)_{n m}$ are scattered complex coefficients, subindices $m$ and $n$ are the modes representing the angular and depth variation of the scattered potential respectively, $d$ is the water depth, $\left(z_{j}, r_{j}, \theta_{j}\right)$ are the cylindrical coordinates local to body $j$ and $k_{0}$ and $k_{n}$ are positive real quantities that represent the progressive and evanescent wave numbers obtained respectively from the following dispersion equations:

$$
k_{0} \tanh k_{0} d=\frac{\omega^{2}}{g} ; \quad k_{n} \tan k_{n} d=-\frac{\omega^{2}}{g}
$$

where $g$ is the acceleration due to gravity.

The expression of the radiated potential follows as in (2) with the only difference being the substitution of the scattered coefficients $\left(A_{j}^{S}\right)_{n m}$ by the 
radiated coefficients $\left(R_{j}^{k}\right)_{n m}$ in a $k$ th mode of motion with $k=1, \ldots, 6$ in the general case:

$$
\begin{aligned}
\phi_{j}^{R, k}=\sum_{m=-\infty}^{\infty}\left[\left(R_{j}^{k}\right)_{0 m} \frac{\cosh k_{0}\left(z_{j}+d\right)}{\cosh k_{0} d} H_{m}^{(1)}\left(k_{0} r_{j}\right)\right. \\
\left.+\sum_{n=1}^{\infty}\left(R_{j}^{k}\right)_{n m} \cos k_{n}\left(z_{j}+d\right) K_{m}\left(k_{n} r_{j}\right)\right] e^{i m \theta_{j}}
\end{aligned}
$$

where $\phi_{j}^{R, k}$ is the radiated potential by a body $j$ moving in its $k$ th degree of freedom.

The incident potential to a body $j$ is represented following the incoming wave solution to the BVP in cylindrical coordinates (a full derivation can be found in Child (2011) 3.4.2):

$$
\begin{aligned}
\phi_{j}^{I}=\sum_{q=-\infty}^{\infty}\left[\left(A_{j}^{I}\right)_{0 q} \frac{\cosh \left[k_{0}\left(z_{j}+d\right)\right]}{\cosh k_{0} d} J_{q}\left(k_{0} r_{j}\right)+\right. \\
\left.\sum_{l=1}^{\infty}\left(A_{j}^{I}\right)_{l q} \cos \left[k_{l}\left(z_{j}+d\right)\right] I_{q}\left(k_{l} r_{j}\right)\right] e^{i q \theta_{j}}
\end{aligned}
$$

where $J_{q}$ is the Bessel function of the first kind of order $q, I_{q}$ is the modified Bessel function of the first kind of order $q,\left(A_{j}^{I}\right)_{l q}$ are incident complex coefficients, subindices $q$ and $l$ are the modes representing the angular and depth variation of the incident potential respectively.

For convenience, 22), (4) and (5) can be represented as the scalar product between a vector of complex coefficients and a vector of partial cylindrical wave components:

$$
\phi_{j}^{S}=\left(A_{j}^{S}\right)^{T} \psi_{j}^{S} ; \quad \phi_{j}^{R, k}=\left(R_{j}^{k}\right)^{T} \psi_{j}^{S} ; \quad \phi_{j}^{I}=\left(A_{j}^{I}\right)^{T} \psi_{j}^{I}
$$

where $T$ stands for transposed, $A_{j}^{S}, A_{j}^{I}$ and $R_{j}^{k}$ are the complex scattered, incident and radiated vectors of partial waves coefficients whose elements represent an $(n, m)$ or $(l, q)$ index pair. For clarity of notation, indexes $(n, m)$ are associated with outgoing waves and $(l, q)$ with incident waves. $\psi_{j}^{S}$ and $\psi_{j}^{I}$ are the 
vectors of scattered and incident cylindrical functions which are expressed as:

$$
\begin{gathered}
\left(\psi_{j}^{S}\right)_{n m}= \begin{cases}\frac{\cosh \left[k_{0}\left(z_{j}+d\right)\right]}{\cosh k_{0} d} H_{m}^{(1)}\left(k_{0} r_{j}\right) e^{i m \theta_{j}} & n=0 \\
\cos \left[k_{n}\left(z_{j}+d\right)\right] K_{m}\left(k_{n} r_{j}\right) e^{i m \theta_{j}} & n \geq 1\end{cases} \\
\left(\psi_{j}^{I}\right)_{l q}= \begin{cases}\frac{\cosh \left[k_{0}\left(z_{j}+d\right)\right]}{\cosh k_{0} d} J_{q}\left(k_{0} r_{j}\right) e^{i q \theta_{j}}, & l=0 \\
\cos \left[k_{l}\left(z_{j}+d\right)\right] I_{q}\left(k_{l} r_{j}\right) e^{i q \theta_{j}}, & l \geq 1\end{cases}
\end{gathered}
$$

The potentials in (6) are expressed in a cylindrical coordinate system $\left(r_{j}, \theta_{j}, \zeta_{j}\right)$ centered in $O_{j} x_{j} y_{j}$ local to each body of the array (Figure 2). They represent a superposition of partial cylindrical waves which are more suited to express the disturbances on the wave field caused by the finite-sized floating bodies than the conventional long-crested plane waves (McNatt J. C. et al., 2013). The velocity potential of a long-crested plane wave is:

$$
\phi_{a}(x, z)=-\frac{i g \zeta_{a}}{\omega} \frac{\cosh k(z+d)}{\cosh k d} e^{i k_{0}(x \cos \beta+y \sin \beta)}
$$

where $\zeta_{a}$ is the wave amplitude, and $\beta$ is the wave direction relative to the global $\mathrm{x}$ axis.

By means of the following fundamental property of the Bessel functions (Watson, 1966, p.14):

$$
e^{\frac{1}{2} z\left(t-\frac{1}{t}\right)}=\sum_{q=-\infty}^{\infty} t^{q} J_{q}(z)
$$

equation (9) can also be expressed as a summation of partial cylindrical waves incident to body $j$ in the form of equation (5) with the following coefficients:

$$
\left(a_{j}^{I}\right)_{l q}= \begin{cases}-i \frac{g \zeta_{a}}{\omega} e^{i k_{0}\left(X_{j} \cos \beta+Y_{j} \sin \beta\right)} i^{q} e^{-i q \beta}, & l=0 \\ 0 & l \geq 1\end{cases}
$$

with $X_{j}$ and $Y_{j}$ the coordinates of the center of body $j$ in the global Cartesian reference system OXY.

Even though the vectors in (6) are theoretically infinitely long, for practical computations they need to be truncated. Their dimension is given as $(2 M+$ $1) \cdot(N+1)$ where the summations go from $m=-M$ to $M$ and from $n=0$ 


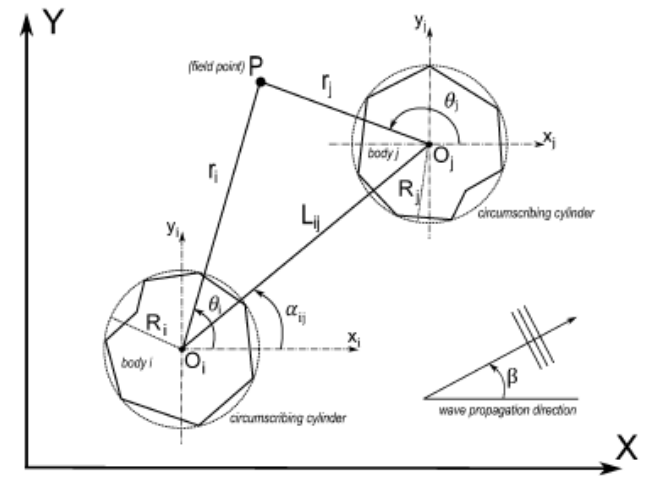

Figure 2: Schematic of the plane view of two bodies of arbitrary geometry with the nomenclature and reference systems used in this paper.

to $N$ (the same expression will apply for modes $q$ and $l$ with truncation limits $Q$ and $L$ respectively). Further details on the derivation of cylindrical partial waves can be found in (Child, 2011; McNatt J. C. et al., 2013).

\subsection{Calculation of the scattering coefficients}

Section 2.1 dealt with the representation of the velocity potential for a single body $j$ (Figure 2) using partial cylindrical waves. We now seek to solve the multiple scattering problem to obtain the scattered coefficients $\left(A_{j}^{S}\right)_{n m}$ for an array of bodies. The analysis follows Kagemoto H. and Yue (1986) and Chakrabarti (2001) and the diffraction and radiation problems are treated separately as in McNatt J. C. et al. (2015).

The total incident potential on body $j$, which can be expressed in the general form $\phi_{j}^{I}=\left(A_{j}^{I}\right)^{T} \psi_{j}^{I}$, is composed of a primary wave (either an ambient plane wave for a diffraction problem or a radiated wave by a body $i$ moving in its $k$ th degree of freedom for a radiation problem) as well as of the unknown scattered potentials from all the neighbouring bodies $i$. For the diffraction problem, the total incident potential on body $j$ reads:

$$
\phi_{j}^{I}=\left(\left(a_{j}^{I}\right)^{T}+\sum_{\substack{i=1 \\ i \neq j}}^{N_{b}}\left(A_{i}^{S}\right)^{T} \mathbf{T}_{i j}\right) \psi_{j}^{I}
$$


where $A_{i}^{S}$ are the unknown scattered coefficients, $a_{j}^{I}$ are the ambient partial cylindrical wave coefficients shown in equation (11) and $\mathbf{T}_{i j}$ is a transformation matrix derived from Graf's addition theorem for Bessel functions (Abramowitz M. and Segun A. 1964). It enables one to express scattered waves from a body $i$ as incident to body $j$ in its local reference system, i.e. $\psi_{i}^{S}=\mathbf{T}_{i j} \psi_{j}^{I}$, and its elements are calculated as:

$$
\left(\mathbf{T}_{i j}\right)_{n n}^{m q}= \begin{cases}H_{m-q}\left(k_{0} L_{i j}\right) e^{i \alpha_{i j}(m-q)} & n=0 \\ K_{m-q}\left(k_{n} L_{i j}\right)(-1)^{q} e^{i \alpha_{i j}(m-q)} & n \geq 1\end{cases}
$$

where $L_{i j}$ is the distance between the centers of bodies $i$ and $j$ and $\alpha_{i j}$ is the angle between body $i$ and body $j$ (see Figure 2).

For the radiation problem, the total incident potential on body $j$ can be expressed as:

$$
\phi_{j}^{I}=\left(\left(a_{j}^{R, i k}\right)^{T}+\sum_{\substack{p=1 \\ p \neq j}}^{N_{b}}\left(A_{p}^{S}\right)^{T} \mathbf{T}_{p j}\right) \psi_{j}^{I}
$$

where $a_{j}^{R, i k}$ are the cylindrical coefficients of the radiated wave incident on body $j$ and generated by the unitary motion of body $i$ in its $k$ th degree of freedom:

$$
\left(a_{j}^{R, i k}\right)= \begin{cases}0 & i=j \\ \mathbf{T}_{i j}^{T} R_{i}^{k} & i \neq j\end{cases}
$$

The incident and scattered partial waves coefficients from an isolated body can be related by means of a linear operator known as Diffraction Transfer $\operatorname{Matrix}\left(\mathbf{B}_{j}\right)$ :

$$
A_{j}^{S}=\mathbf{B}_{j} A_{j}^{I}
$$

The elements $\left(\mathbf{B}_{j}\right)_{n l}^{m q}$ in row $(n, m)$ and column $(l, q)$ are defined as the coefficient of the partial wave of depth mode $n$ and angular mode $m$ in the scattered potential in response to a unit incident wave of depth mode $l$ and angular mode $q$ (Kagemoto H. and Yue, 1986). For the diffraction problem, by substituting the incident partial coefficients from 12 into 16 , the scattered 
coefficients of body $j$ read:

$$
A_{j}^{S}=\mathbf{B}_{j}\left(a_{j}^{I}+\sum_{\substack{i=1 \\ i \neq j}}^{N_{b}} \mathbf{T}_{i j}^{T} A_{i}^{S}\right)
$$

For the radiation problem, the substitution of the incident partial coefficients from (14) into (16) leads to the scattered coefficients of body $j$ :

$$
A_{j}^{S}=\mathbf{B}_{j}\left(a_{j}^{R, i k}+\sum_{\substack{p=1 \\ p \neq j}}^{N_{b}} \mathbf{T}_{p j}^{T} A_{p}^{S}\right)
$$

Considering all the bodies in the array, equation (17) for the diffraction problem, or 18) for the radiation problem, become a system of equations which can be solved for all the unknown scattered coefficients:

$$
A^{S}=(\mathbf{I}-\mathbf{B T})^{-1} \mathbf{B} a^{I}
$$

where $\mathbf{I}$ is the identity matrix, $a^{I}$ is a vector containing the partial coefficients of the incident primary waves (either ambient plane waves for the diffraction problem or radiated waves by a body $i$ moving in its $k$ th degree of freedom for a radiation problem) and $\mathbf{B}$ and $\mathbf{T}$ are matrices containing respectively the diffraction transfer matrices and transformation matrices of the bodies in the array organised in an appropriate manner. In equation 190 the matrices are square; their dimensions and the lengths of the column vectors are $(2 M+1)$. $(N+1) \cdot N_{b}$. The term $(\mathbf{I}-\mathbf{B T})^{-1}$ is usually referred to as the scattering matrix (Siddorn P. and Eatock Taylor, 2008).

Before equation (17) can be solved, one must determine the DTM $\left(\mathbf{B}_{j}\right)$. In case of equation 18 , the RC $\left(R_{j}\right)$ must also be determined. Both operators are calculated with the body in isolation and depend on its geometry and the wave frequency $(\omega)$. In addition, the RC depend on the mode of motion. Two methodologies to compute them (Goo, J.-S. and Yoshida, 1990; McNatt J. C. et al. 2015) are presented in this paper in section 3 The former has been implemented in BEM code NEMOH and will be referred to as Method I, the latter serves as a basis of comparison for Method $I$ and shall be referred to as Method II. 
2.3. Calculation of the radiation hydrodynamic coefficients and the excitation forces

The solution to the multiple scattering problem (19) enables one to reconstruct the total potential in the fluid domain and to compute the forces exerted on the bodies. McNatt J. C. et al. (2015) introduced a linear operator called Force Transfer Matrix $\left(\mathbf{G}_{j}\right)$ which relates the forces acting in each degree of freedom of the body to the partial incident cylindrical wave coefficients. Here we make use of it to compute the excitation forces as:

$$
F_{j}^{E}=\mathbf{G}_{j} A_{j}^{I}
$$

where $F_{j}^{E}$ is the vector of excitation forces with dimension $D f_{j}$ and $A_{j}^{I}$ are the cylindrical coefficients of the total wave incident to body $j$ composed of the ambient incident wave and all the scattered waves of neighbouring bodies:

$$
A_{j}^{I}=\left(a_{j}^{I}+\sum_{\substack{i=1 \\ i \neq j}}^{N} \mathbf{T}_{i j}^{T} A_{i}^{S}\right)
$$

Similarly, following the procedure by McNatt J. C. et al. (2015), the radiation force can be computed as :

$$
\left(F_{j}^{R}\right)^{i k}= \begin{cases}\mathbf{G}_{j} A_{j}^{I^{\prime}} & j \neq i \\ \left(i \omega\left(\mathbf{A} \mathbf{M}_{j}\right)^{k}-\left(\mathbf{D}_{j}\right)^{k}\right)+\mathbf{G}_{j} A_{j}^{I^{\prime}} & j=i\end{cases}
$$

where $\left(F_{j}^{R}\right)^{i k}$ is the vector of radiation forces on body $j$ due to a motion of unit amplitude of body $i$ in a degree of freedom $k,\left(\mathbf{A} \mathbf{M}_{j}\right)^{k}$ is the $k^{t h}$ column of the added mass matrix of the isolated body $j,\left(\mathbf{D}_{j}\right)^{k}$ is the $k^{t h}$ column of the radiation damping matrix of the isolated body $j$ and $A_{j}^{I^{\prime}}$ are the cylindrical coefficients of the total wave incident to body $j$ composed of the radiated wave cylindrical coefficients $a_{j}^{R, i k}$ generated by the motion of body $i$ in the degree of freedom $k$ plus all the scattered waves by the fixed neighbouring bodies:

$$
A_{j}^{I^{\prime}}=\left(a_{j}^{R, i k}+\sum_{\substack{p=1 \\ p \neq j}}^{N} \mathbf{T}_{p j}^{T} A_{p}^{S}\right)
$$


The dimension of the added mass and radiation damping hydrodynamic

\section{Hydrodynamic operators}

\subsection{Method I}

\subsubsection{Diffraction Transfer Matrix}

The methodology developed by Goo, J.-S. and Yoshida (1990) enables one to find each element of the diffraction transfer matrix $\left(\mathbf{B}_{j}\right)_{n l}^{m q}$ of an isolated body $j$ following two steps. First, the solution to a diffraction problem where the incident wave is a cylindrical partial wave of angular mode $q$ and depth mode $l$ is found. This involves solving for the unknown source strengths $\sigma_{l q j}$ in the following integral equation:

$$
\begin{aligned}
& \frac{1}{2} \sigma_{l q j}\left(r_{j}, \theta_{j}, z_{j}\right)+ \\
& \qquad \int_{S_{j}} \sigma_{l q j}\left(R_{j}, \Theta_{j}, \zeta_{j}\right) \frac{\partial G_{j}\left(R_{j}, \Theta_{j}, \zeta_{j} ; r_{j}, \theta_{j}, z_{j}\right)}{\partial n} d s \\
& =-\frac{\partial\left(\psi_{j}^{I}\right)_{l q}\left(r_{j}, \theta_{j}, z_{j}\right)}{\partial n}
\end{aligned}
$$

where $G$ is the Green's function in finite depth, $\left(R_{j}, \Theta_{j}, \zeta_{j}\right)$ is the influencing or source point and $\left(r_{j}, \theta_{j}, z_{j}\right)$ the influenced or field point, $\left(\psi_{j}^{I}\right)_{l q}$ is the incident partial wave $l q$ as defined in $(8)$ and $S_{j}$ the wetted surface of body $j$.

The right hand side of expression (24) expresses the diffraction boundary condition on the wetted surface of the body due to an incident partial cylindrical wave of angular mode $q$ and depth mode $l$. This boundary condition must 
replace the standard diffraction boundary condition in BEM codes where the incident potential is simply the potential of a planar wave.

Once the source strength distribution $\sigma_{l q j}$ is known on the panelized surface of the body, the following step consists of expressing the scattered potential in the base of partial wave functions (2). This is to be achieved by making use of the Green's function in cylindrical coordinates derived by Fenton (1978) which leads to the following expressions for the elements of the Diffraction Transfer Matrix:

$$
\begin{aligned}
\left(\mathbf{B}_{j}\right)_{0 l}^{m q}= & \frac{i}{2} \frac{K^{2}-k_{0}^{2}}{\left(k_{0}^{2}-K^{2}\right) d+K} \cosh k_{0} d \times \\
& \iint_{S_{j}} \sigma_{l q j}\left(R_{j}, \Theta_{j}, \zeta_{j}\right) J_{m}\left(k_{0} R_{j}\right) \cosh \left[k_{0}\left(\zeta_{j}+d\right)\right] e^{-i m \Theta_{j}} d s \\
\left(\mathbf{B}_{j}\right)_{n l}^{m q}=-\frac{1}{\pi} & \frac{k_{n}^{2}+K^{2}}{\left(k_{n}^{2}+K^{2}\right) d-K} \times \\
& \iint_{S_{j}} \sigma_{l q j}\left(R_{j}, \Theta_{j}, \zeta_{j}\right) I_{m}\left(k_{n} R_{j}\right) \cos \left[k_{n}\left(\zeta_{j}+d\right)\right] e^{-i m \Theta_{j}} d s
\end{aligned}
$$

where $K=\omega^{2} / g$.

\subsubsection{Radiation Characteristics}

The same principle applied for the calculation of the DTM can be used to obtain the RC vector. First, the radiation problem associated with a degree of freedom $k$ of the body under consideration is solved. In this case, the boundaryvalue problem is the same as the one solved by a standard BEM solver. Thus, the source strength distribution can be obtained by solving the following equation:

$$
\begin{aligned}
& \frac{1}{2} \sigma_{j k}\left(r_{j}, \theta_{j}, z_{j}\right)+ \\
& \iint_{S_{j}} \sigma_{j k}\left(R_{j}, \Theta_{j}, \zeta_{j}\right) \frac{\partial G_{j}\left(R_{j}, \Theta_{j}, \zeta_{j} ; r_{j}, \theta_{j}, z_{j}\right)}{\partial n} d s_{j}=n_{j k}
\end{aligned}
$$

where $n_{j k}$ is the generalized direction cosine on the immersed surface of body $j$ and describes the distribution of normal velocities due to a unitary motion on the $k$ th degree of freedom. 
Once known, the source strength distribution $\sigma_{j k}$ is used in conjunction with the Green's function in cylindrical coordinates to express the radiated potential in terms of partial waves leading to expressions for the radiation characteristics:

$$
\begin{aligned}
\left(R_{j}^{k}\right)_{0 m}= & \frac{i}{2} \frac{K^{2}-k_{0}^{2}}{\left(k_{0}^{2}-K^{2}\right) d+K} \cosh k_{0} d \times \\
& \iint_{S_{j}} \sigma_{j k}\left(R_{j}, \Theta_{j}, \zeta_{j}\right) J_{m}\left(k_{0} R_{j}\right) \cosh \left[k_{0}\left(\zeta_{j}+d\right)\right] e^{-i m \Theta_{j}} d s \\
\left(R_{j}^{k}\right)_{n m}=-\frac{1}{\pi} & \frac{k_{n}^{2}+K^{2}}{\left(k_{n}^{2}+K^{2}\right) d-K} \times \\
& \iint_{S_{j}} \sigma_{j k}\left(R_{j}, \Theta_{j}, \zeta_{j}\right) I_{m}\left(k_{n} R_{j}\right) \cos \left[k_{n}\left(\zeta_{j}+d\right)\right] e^{-i m \Theta_{j}} d s
\end{aligned}
$$

\subsection{Method II}

\subsubsection{Diffraction Transfer Matrix}

The Method I described in the previous section involves solving the scattering BVP using incident partial cylindrical waves. To circumvent the need to use incident partial cylindrical waves, a procedure to find the DTM by solving the diffraction problem only for incident plane waves was described in McNatt J.

180 C. et al. (2015). Equation (16) can be transformed into a system of equations to solve for the elements of the DTM as long as a large enough number of pairs of scattered/incident vectors of coefficients is known in advance. This is to be achieved by solving, at least, as many plane wave diffraction problems as the dimension of the DTM by changing at each time the propagation direction $(\beta)$ of the plane incident wave. The vectors of incident partial waves are known from an analytical expression (11) whereas the elements of the associated vectors of the scattered coefficients can be derived by means of a Fourier transform of the scattered potential on the body circumscribing cylinder:

$$
\begin{aligned}
a_{0 m}^{S}=-\frac{i}{2 \pi} \frac{\omega}{g} \frac{2 \cosh k_{0} d}{d\left(1+\frac{\sinh 2 k_{0} d}{2 k_{0} d}\right)} \frac{1}{H_{m}^{(2)}\left(k_{0} r_{0}\right)} \times \\
\int_{-d}^{0} \int_{0}^{2 \pi} \phi\left(r_{0}, \theta, z\right) e^{-i m \theta} \cosh k_{0}(d+z) d \theta d z
\end{aligned}
$$




$$
\begin{aligned}
b_{n m}^{S}=-\frac{i}{2 \pi} \frac{\omega}{g} \frac{2}{d\left(1+\frac{\sin 2 k_{n} d}{2 k_{n} d}\right)} \frac{1}{K_{m}\left(k_{n} r_{0}\right)} \times \\
\int_{-d}^{0} \int_{0}^{2 \pi} \phi\left(r_{0}, \theta, z\right) e^{-i m \theta} \cos k_{n}(d+z) d \theta d z
\end{aligned}
$$

where $\left(r_{0}, \theta, z\right)$ are the coordinates of the points on a circumscribing cylindrical control surface and $a_{0 m}^{S}$ and $b_{n m}^{S}$ are the progressive and evanescent cylindrical wave coefficients which correspond respectively to $A_{0 m}^{S}$ and $A_{n m}^{S}$ of Method I (as detailed in section 3.3.

\subsubsection{Radiation Characteristics}

The computation of the RC follows the same procedure as for the DTM. First, the radiation problem associated with a specific mode of motion and frequency is solved with a standard BEM code. Then, by means of expressions (30) and (31), the radiated potential is expressed in terms of partial cylindrical wave functions. In this base the coefficients are known as Radiation Characterand $R_{n m}^{k}$ in the notation of Method I.

\subsection{Equivalence between Methods I and II}

The formulation of Method $I$ is based on the notation of Goo, J.-S. and Yoshida (1990) which makes use of a negative harmonic time dependence $\left(e^{-i \omega t}\right)$, whereas Method II by McNatt J. C. et al. (2015) adopts a positive sign convention $\left(e^{i \omega t}\right)$. In addition, in Method I amplitudes of partial wave coefficients have units of velocity potential $\left(\mathrm{m}^{2} / \mathrm{s}\right)$. In Method II they have units of length (m) and get units of velocity potential by multiplying by $\left(i \frac{g}{\omega}\right)$. By taking into account such convention differences, the equivalence between both partial wave coefficients is given by:

$$
(-1)^{-m} \frac{g}{\omega}\left[i\left(a_{-m}^{S, I}\right)\right]^{*}=A_{m}^{S, I}
$$


where $^{*}$ denotes complex conjugate, $a_{-m}^{S, I}$ are the progressive partial waves coefficients in the notation of McNatt J. C. et al. (2015) and $A_{m}^{S, I}$ in the notation of Goo, J.-S. and Yoshida (1990).

By introducing (32) into the definition of the DTM in (16), the following expression relating their elements in both notations can be obtained:

$$
\frac{(-1)^{-m}}{(-1)^{-q}}\left(\mathbf{B}^{*}\right)_{-m,-q}^{M e t h o d ~ I}=(\mathbf{B})_{m, q}^{\text {Method II }}
$$

The results presented in this paper have been computed by using the BEM solver NEMOH, in which Method I has been implemented. The results presented for Method II were computed using WAMIT. To solve the radiation problem, NEMOH employs unit-amplitude velocity as the boundary condition, while WAMIT uses unit-amplitude motions, which results in a difference in scaling of the resulting solutions. The relationship between the source strengths in the radiation problem is:

$$
\sigma^{N E M O H}=\frac{1}{i \omega} \sigma^{W A M I T}
$$

As a consequence, by taking into account (34) and the notation convention differences in (32), the relationship between the Radiation Characteristics (RC) in both notations follows as:

$$
(-1)^{-m} \frac{g}{\omega^{2}}\left[\left(a_{-m k}^{R}\right)\right]^{*}=R_{m k}
$$

where $a_{-m k}^{R}$ are the RC in the notation of McNatt J. C. et al. (2015) and $R_{m k}$ in the notation of Goo, J.-S. and Yoshida (1990).

The expressions of the scattered and radiated potentials expressed on the basis of partial cylindrical waves are only valid outside the body's circumscribing cylinder of radius $R_{j}$. For Method $I$, the limitation stems from the use of the cylindrical Green's function form by Fenton (1978) valid only when $r_{j}>R_{j}$ whereas, for Method II, it is a consequence of performing a Fourier Transform of the potential on the body's circumscribing cylinder. Because of that, the use of the interaction theory requires that the relative distance between two bodies in the array has to be such that a circumscribing cylinder cannot intersect any other 
body (see Figure 3 in Chakrabarti (2001)). This condition is more restrictive than the one imposed by the use of Graf's addition theorem in 13 which states that the circumscribing cylinder of a body cannot contain the geometrical center of any other body. In that sense, we note that the arrangement in Figure 5b by McNatt J. C. et al. (2015) is technically not in the range of validity of the interaction theory.

Even if evanescent terms from the scattered potential can be identified using (31), the use of only plane progressive incident waves (with no evanescent components) prevents the calculation of the DTM terms of the scattering due to incident evanescent waves using Method II.

\section{Numerical Implementation}

As mentioned in Babarit A. and Delhommeau (2015), the body boundary conditions in the BEM solver NEMOH can be defined by the user. Thus, for the diffraction problem, the code can easily accommodate a user-defined distribution of normal velocities at the centroid of each mesh panel. They have been implemented as the derivative of the incident partial wave functions (of angular mode $q$ and depth mode $l$ ) represented on the right hand side of (24) and whose full expression can be found in Goo, J.-S. and Yoshida (1990). The finite depth Green's function is expressed in Cartesian coordinates, rather than the cylindrical coordinates of equation (24) as it was more convenient for the computations.

The term $\frac{K^{2}-k_{0}^{2}}{\left(k_{0}^{2}-K^{2}\right) d+K}$ in expressions 25) and 28 poses convergence problems when the water depth is increased. As mentioned in Peter M. A. and Meylan H. (2004), the limitation can be circumvented if it is reformulated by means of the dispersion relation (3). In addition, the condition number of the system (19) has been improved by normalizing Bessel functions with respect to both their order and argument as suggested by Child (2011).

No closed mathematical expression exists for the integrals $[25)-(26)$ and $(28)$ 29 , even when $\sigma$ is constant over each panel. Therefore, they cannot be solved 

As expected, it increases for increasing orders.

\section{Results and Discussion}

In the first part of this section, the components of the DTM and the RC for a truncated vertical cylinder and a square box are presented. Two different discretizations, shown in Figures $4 a$ and $5 a$, have been used for the computations. A mesh convergence study has been performed showing no significant improvement of accuracy by using 43 or 53 . In some cases, the hydrodynamic operators 
a

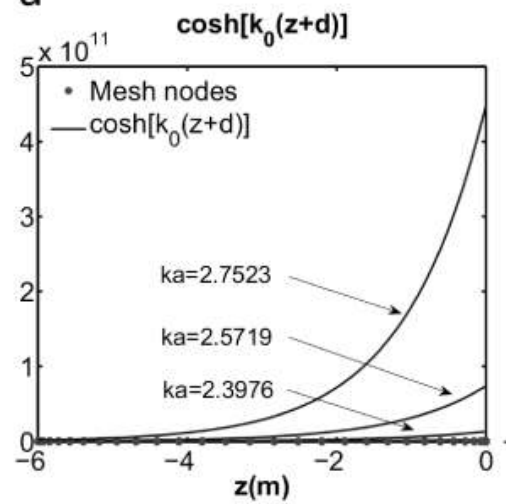

d

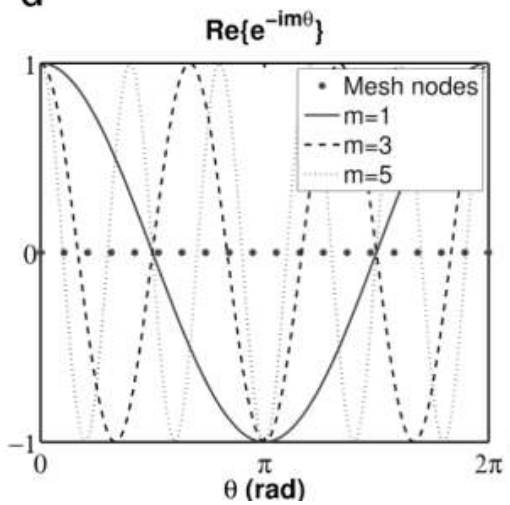

b

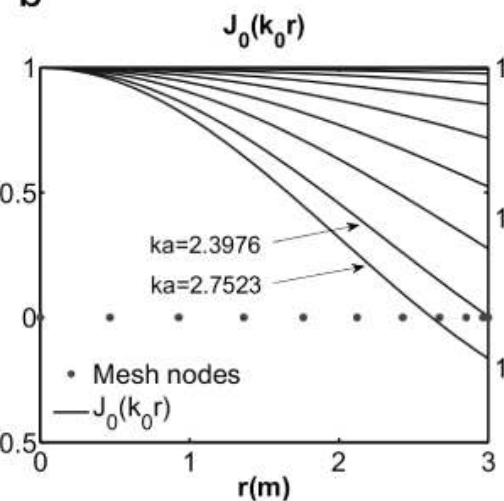

e

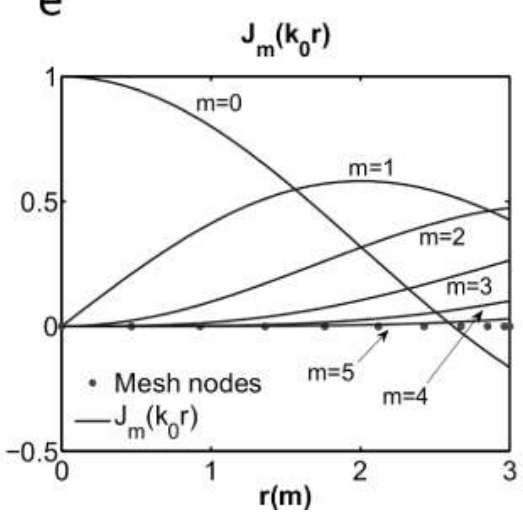

C

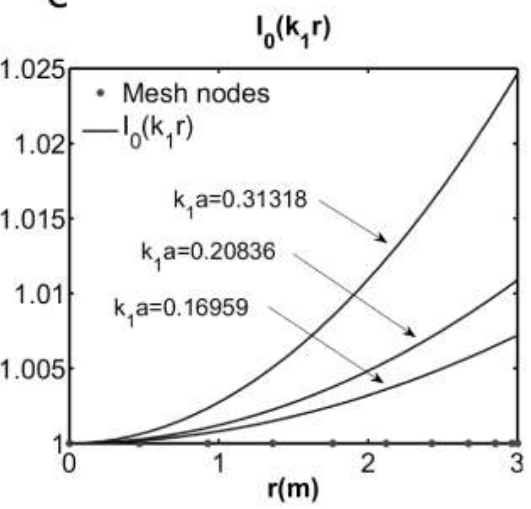

f

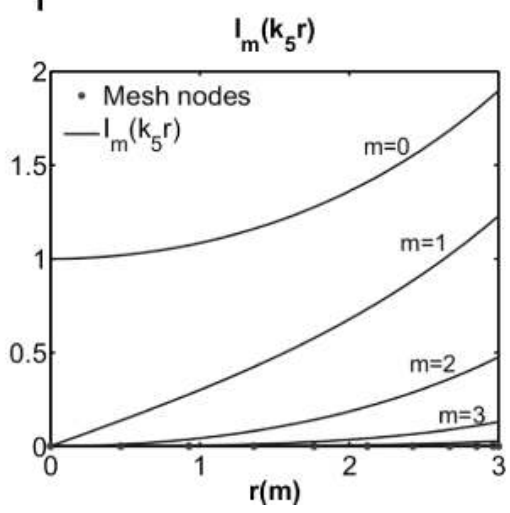

Figure 3: Variation of the hyperbolic cosine depth dependence $(a)$ and angular dependance $(d)$, variation of the Bessel function of the first kind $(b, e)$ and of the modified Bessel function of the first kind $(c, f)$ along the lateral side and the bottom of a cylinder (3m radius, $6 \mathrm{~m}$ draft in a $30 \mathrm{~m}$ water depth). Results in (e) are calculated for $k_{0} a=2.7523$ and in $(f)$ for $k_{5} a=1.7279$. The thick points represent the nodes of a given mesh with cosine spacing. 
a

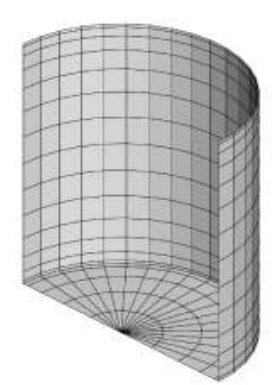

b

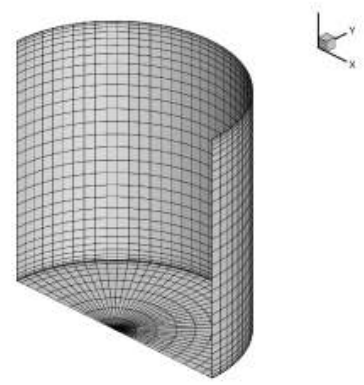

Figure 4: Truncated vertical cylinder mesh. Only half of the geometry is shown due to symmetry. (a) - coarse mesh, 361 panels; (b) - fine mesh 1521 panels.

a

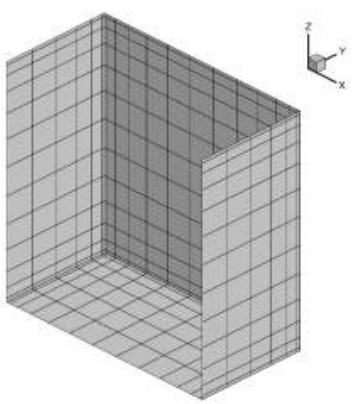

b

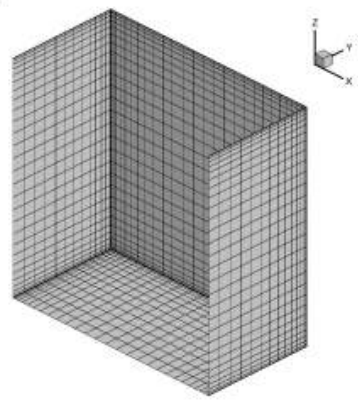

Figure 5: Square box mesh. Only half of the geometry is shown due to symmetry. (a) - coarse mesh, 403 panels; (b) - fine mesh 2059 panels.

obtained with Method $I$ are not shown in the region $2.2<k a<2.6$. This is because of the spurious results that were found at a discrete set of frequencies within this range, known as irregular frequencies, where the boundary value problem is ill-posed and the numerical scheme of the BEM solver NEMOH does not converge to the physical solution.

In the second part, the hydrodynamic coefficients obtained using the interaction theory by Kagemoto H. and Yue (1986) into which the calculated DTM and $\mathrm{RC}$ have been embedded are shown for a small array of two and four truncated vertical cylinders. For the latter, the free surface elevation is also displayed. 
a

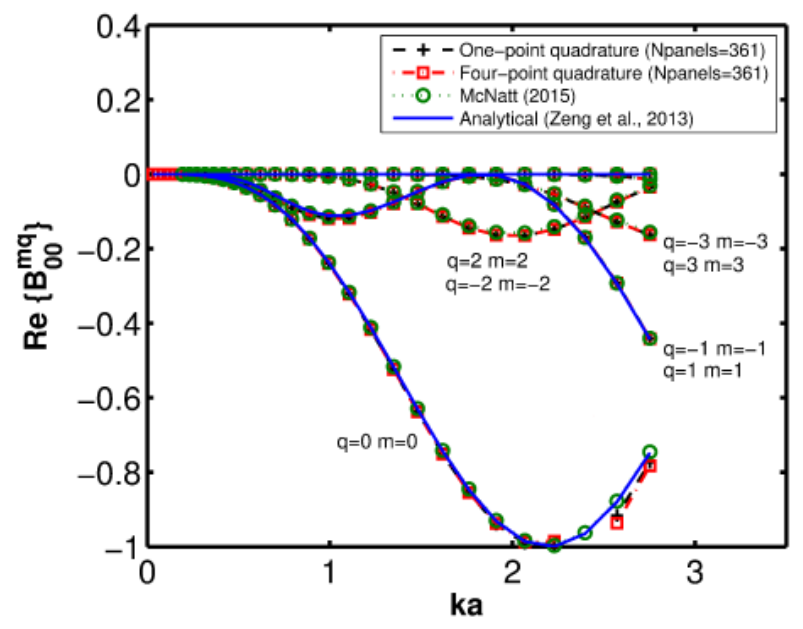

b

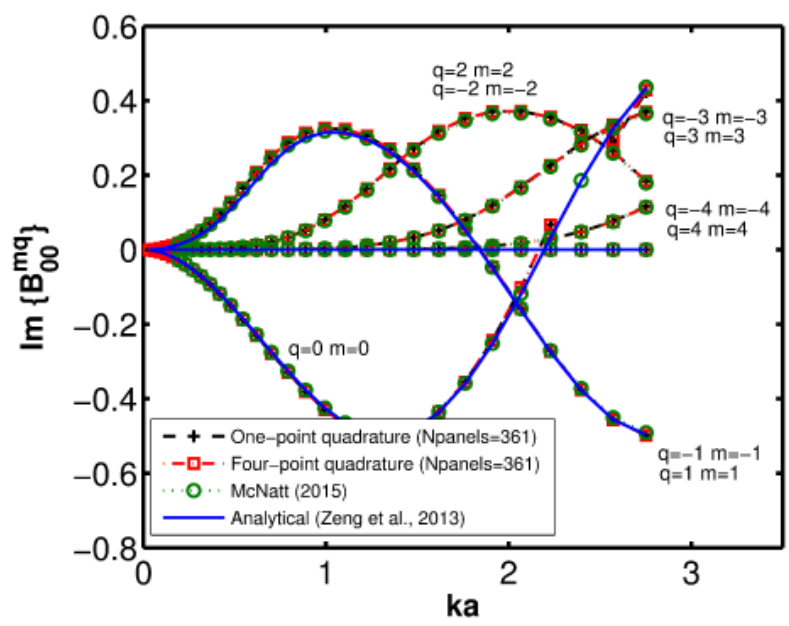

Figure 6: Real and imaginary parts of the Diffraction Transfer Matrix progressive terms for a truncated vertical cylinder of $3 m$ radius $(a), 6 m$ draft in a $10 m$ water depth.

\subsection{Diffraction Transfer Matrix}

\subsubsection{Cylinder}

Figures $6 a$ and 63 show the real and imaginary parts of the cylinder DTM terms. For clarity, only progressive and non-negligible terms are shown and annotated with their respective $(q, m)$ indexes. A very good agreement is found between results obtained using Method I (with both one-point and four-point quadrature schemes), Method II and the semi-analytical solution by Zeng and Tang (2013) which is based on the eigenfunction expansion with $|m|<1$ and the Graf's addition theorem for Bessel functions. It can be observed that the only non-zero DTM terms correspond to pairs of equal incident $(q)$ and outgoing $(m)$ angular modes. This is a particular feature of axisymmetric geometries such

as the truncated vertical cylinder, as was identified by Kagemoto H. and Yue (1986). In addition, it can be appreciated that the number of non-negligible angular modes is frequency dependent. For instance, a truncation of only two angular modes is sufficient at $k a \approx 1$ but not at $k a \approx 2$ where three angular modes are required. 
a

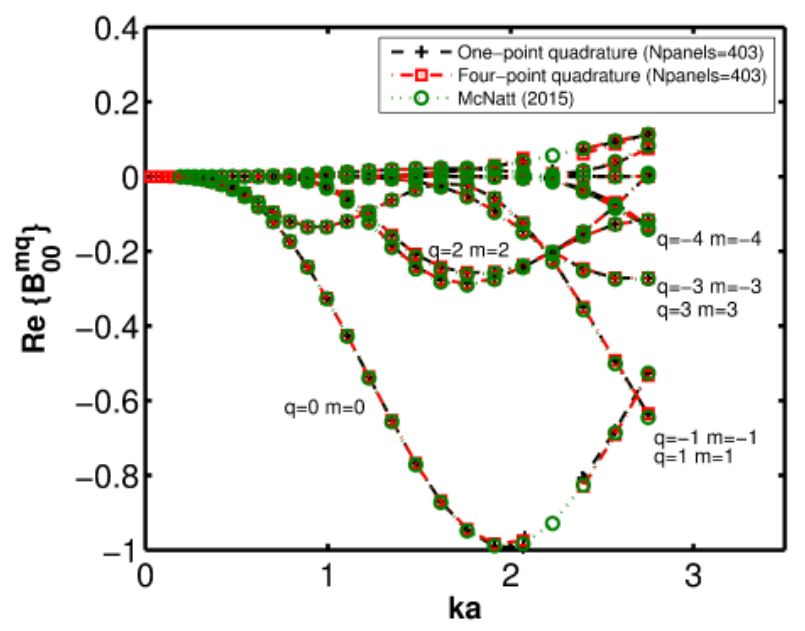

b

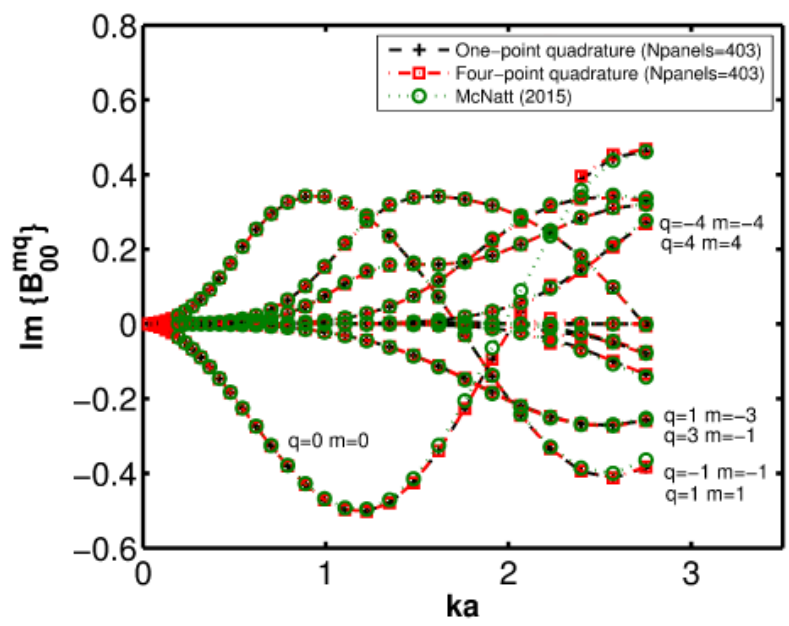

Figure 7: Real and imaginary parts of the Diffraction Transfer Matrix progressive terms for a cube box of $6 m$ side $(2 a), 6 m$ draft in a $10 m$ water depth.

\subsubsection{Square Box}

Figures $7 a$ and $7 b$ show the real and imaginary parts of the square box DTM components. Similar to the truncated vertical cylinder, good agreement between the results obtained using Method $I$ and $I I$ can be observed. For this non-axisymmetric geometry, terms of the DTM involving different incident and outgoing angular modes, such as the pair $(q=1, m=-3)$, are non-zero. In addition, it can be seen that for a wide range of $k a$ values the angular mode truncation is higher than for the truncated vertical cylinder. No improvement of results is obtained for a four point quadrature as compared to a one point quadrature integration scheme.

\subsection{Radiation Characteristics}

\subsubsection{Cylinder}

The progressive terms of the surge Radiation Characteristics of the cylinder are shown in Figure 8. Good agreement between both methods and the semi-analytical solution can be observed. Like with the DTM, there were no 
significant differences between integration schemes. For this mode of motion, it can be observed that only modes $m=1$ and $m=-1$ are non-zero which correspond to the partial wave shown in Figure $1 p$.

For heave (Figure 10, only the mode $m=0$, representing an isotropic wave 320 (Figure 1 $1 a$ ) is required (McNatt J. C. et al., 2013). There is a difference in the magnitude of both the real and the imaginary parts of the $m=0$ wave between the outputs from Method I, Method II, and the semi-analytical results. This discrepancy is attributed to the use of a zeroth-order indirect BEM solver, which does not provide precise results near the sharp corners on the bottom of the cylindrical and the square box geometries where a large variation of the flow velocity occurs. A better agreement with analytical results for the components of the DTM and the RC was shown in Kashiwagi (2000, 2005, 2008) by using a direct and higher-order BEM. It offers several advantages with respect to the indirect BEM, namely that there is no need to evaluate the surface integral on the body surface with the normal derivative of the incident-wave potential for the diffraction problem and that the wave-exciting force can be computed directly using the velocity potential obtained as a solution of the integral equation. The discrepancy between Method $I$ and $I I$ is mainly attributed to differences in the results provided by both NEMOH and WAMIT BEM solvers, as shown for 335 instance by Crooks et al. (2016).

\subsubsection{Square Box}

Compared to the cylinder, additional angular modes of order three appear in the Radiation Characteristics of the square box moving in surge (Figure 9). The radiated field for this geometry and for this mode of motion is more complex than the one generated by the truncated vertical cylinder and a superposition of partial waves is required to represent it. For heave (Figure 11), it is numerically found that only the isotropic partial wave $(m=0)$ is needed and we note a better agreement between Method I and Method II than for the truncated vertical cylinder. 
a

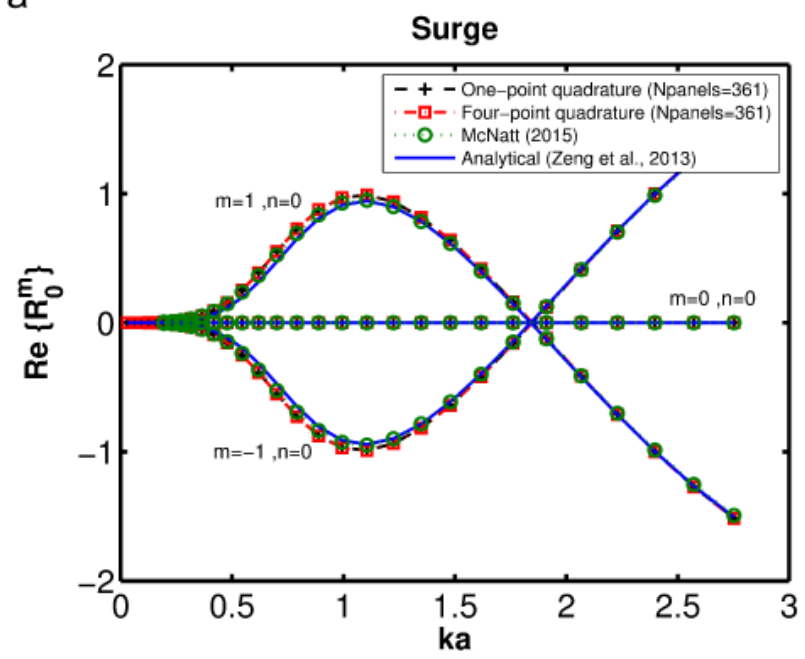

b

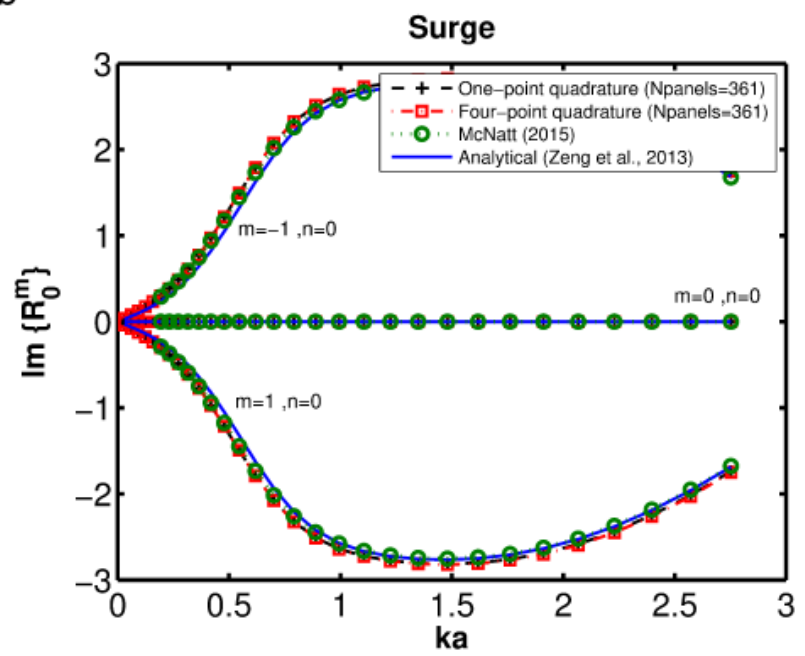

Figure 8: Real and imaginary parts of the Radiation Characteristics progressive terms for a truncated vertical cylinder of $3 m$ radius $(a), 6 m$ draft moving in surge in a $10 m$ water depth.

a

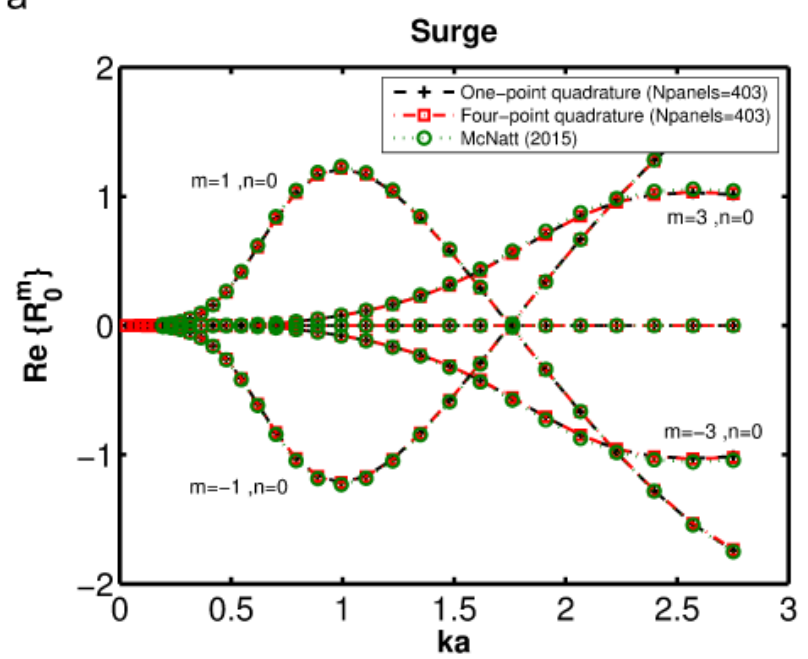

b

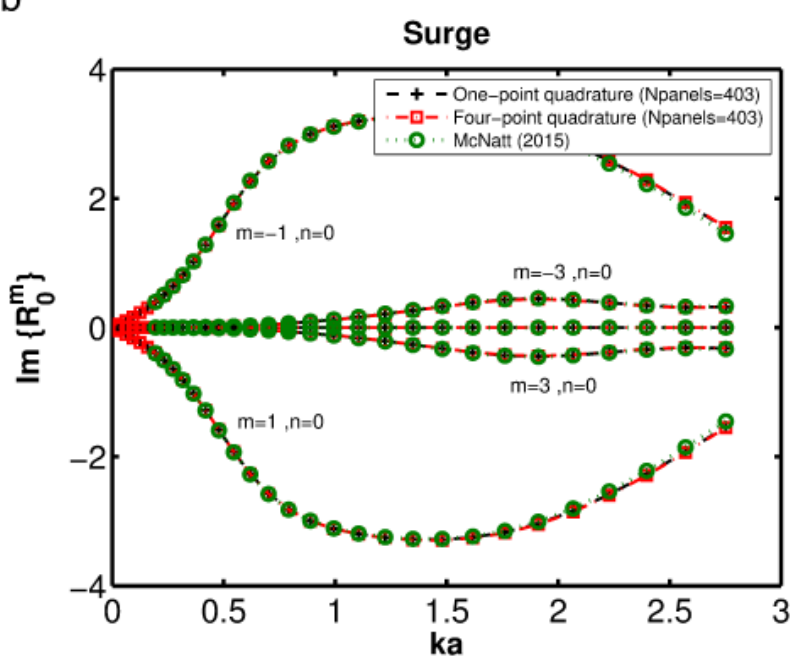

Figure 9: Real and imaginary parts of the Radiation Characteristics progressive terms for a square box of $6 m$ side $(2 a), 6 m$ draft moving in surge in a $10 m$ water depth. 
a

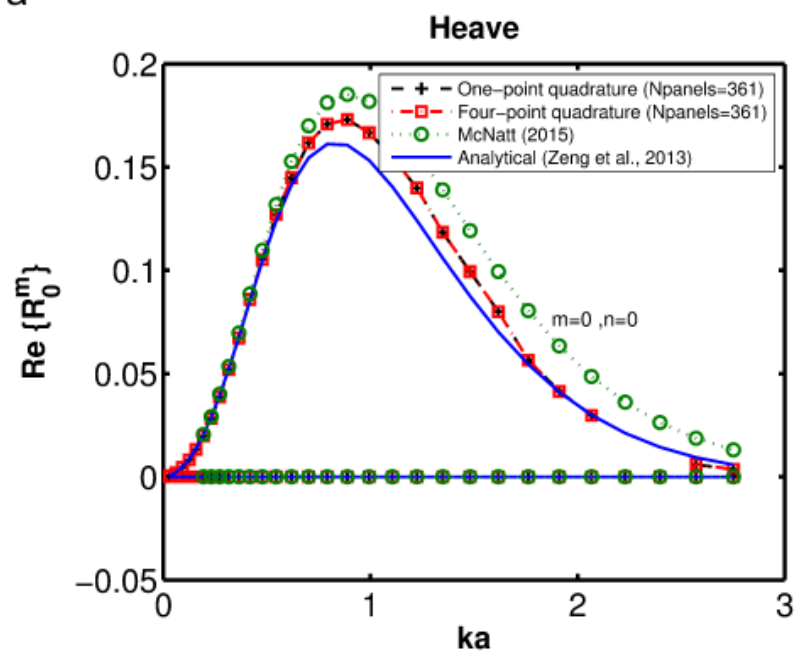

b

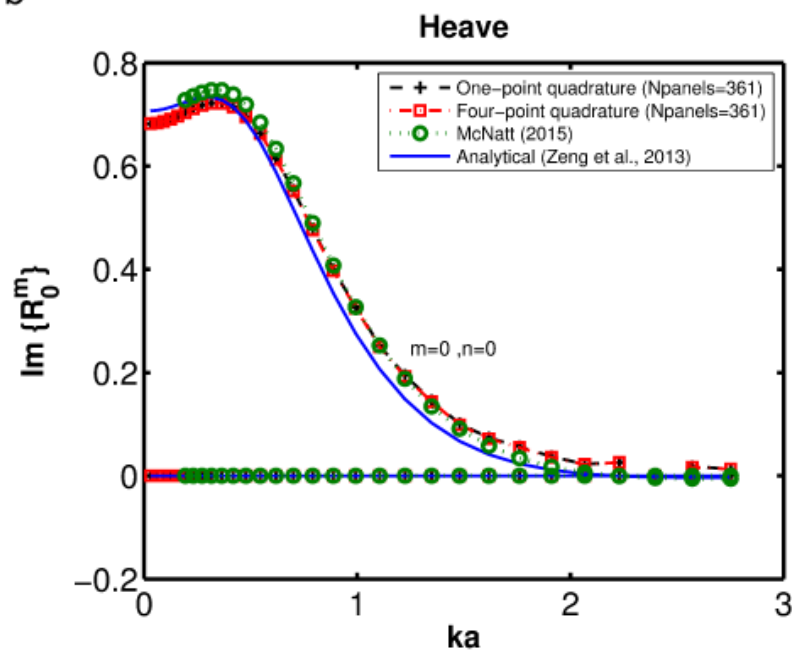

Figure 10: Real and imaginary parts of the Radiation Characteristics progressive terms for a truncated vertical cylinder of $3 \mathrm{~m}$ radius $(a), 6 \mathrm{~m}$ draft moving in heave in a $10 \mathrm{~m}$ water depth.

a

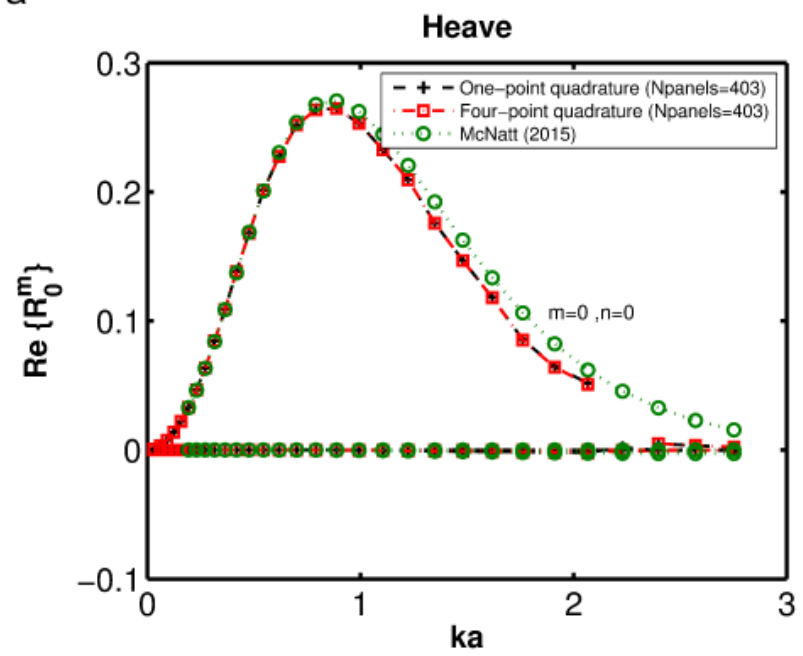

b

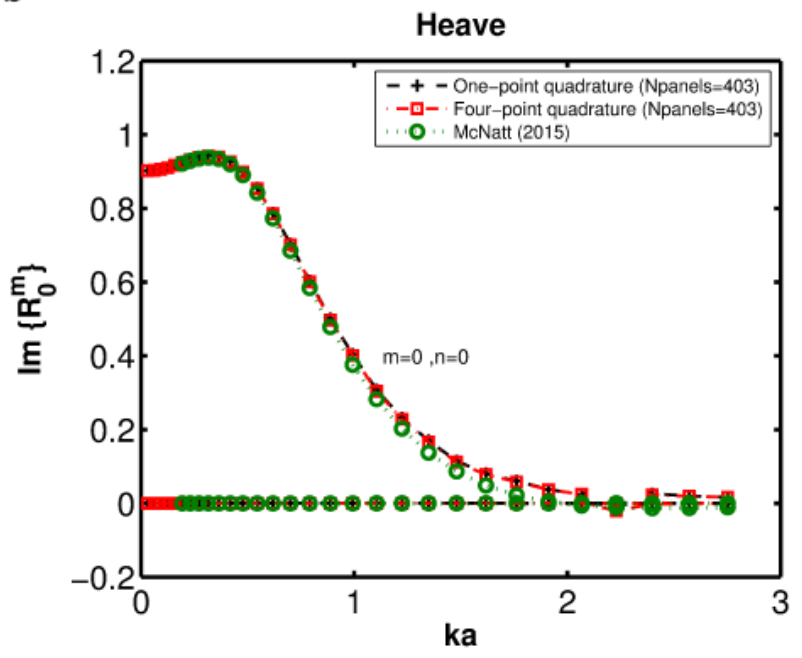

Figure 11: Real and imaginary parts of the Radiation Characteristics progressive terms for a square box of $6 m$ side $(2 a), 6 m$ draft moving in heave in a $10 m$ water depth. 


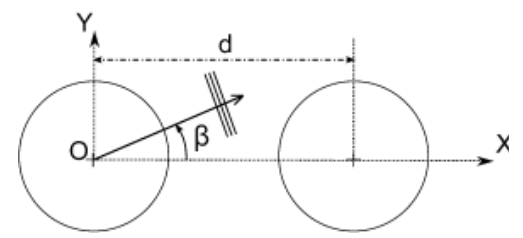

Figure 12: Schematic of an array composed of two truncated vertical cylinders. $\beta=0$ corresponds to the positive $x$ - axis.

a

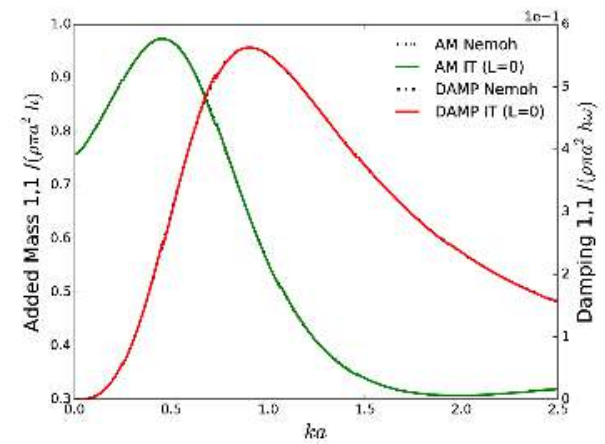

b

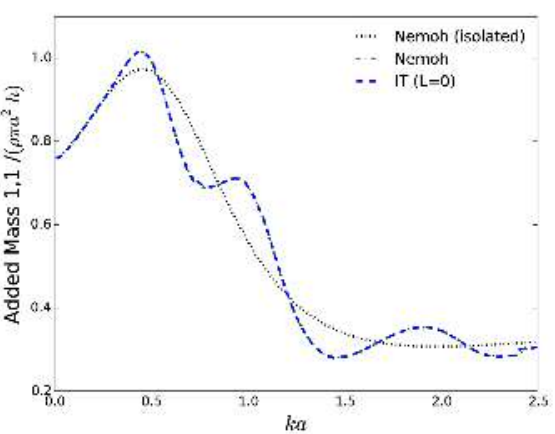

c

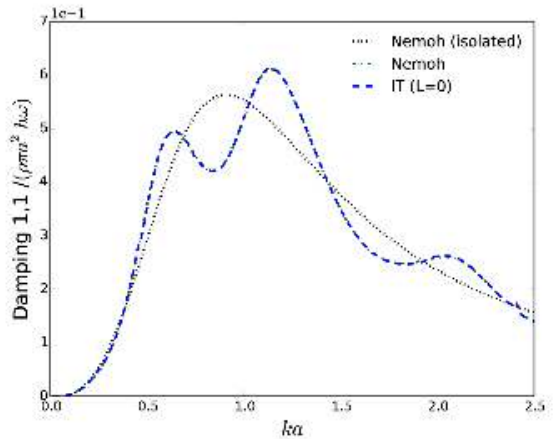

Figure 13: Added mass and damping coefficients for the first truncated vertical cylinder (radius $(a)$, draft $(h=2 a)$ in a water depth $(50 a / 3))$ in the two body array (Figure 12 for different separating distances $(d / a=5000-(a), d / a=5-(b), d / a=5-(c))$. First index corresponds to the direction of the force and the second to the degree of freedom.

\subsection{Influence of the evanescent modes truncation}

a Results for the hydrodynamic coefficients obtained using the IT by Kagemoto H. and Yue (1986) described in section 2 are shown in Figure 13 for a small array of two cylinders of radius $(a)$, draft $(h=2 a)$ in a $50 a / 3$ water depth (Figure 12. . They are compared to direct calculations performed with our BEM code. Hydrodynamic coefficients are specified by means of two indexes; 1-6 are the six degrees of freedom (DOFs) of the first body, and 7-12 are the 6 DOFs of the second one. For instance, added mass (3-9) is the added mass of heave-heave coupling between the bodies. Several separating distance cases are considered.

355 When only a single line is visible, it means that the other lines lie underneath it and there is a very good match between results.

First, both bodies are set far away from each other at a distance $d / a=5000$. 


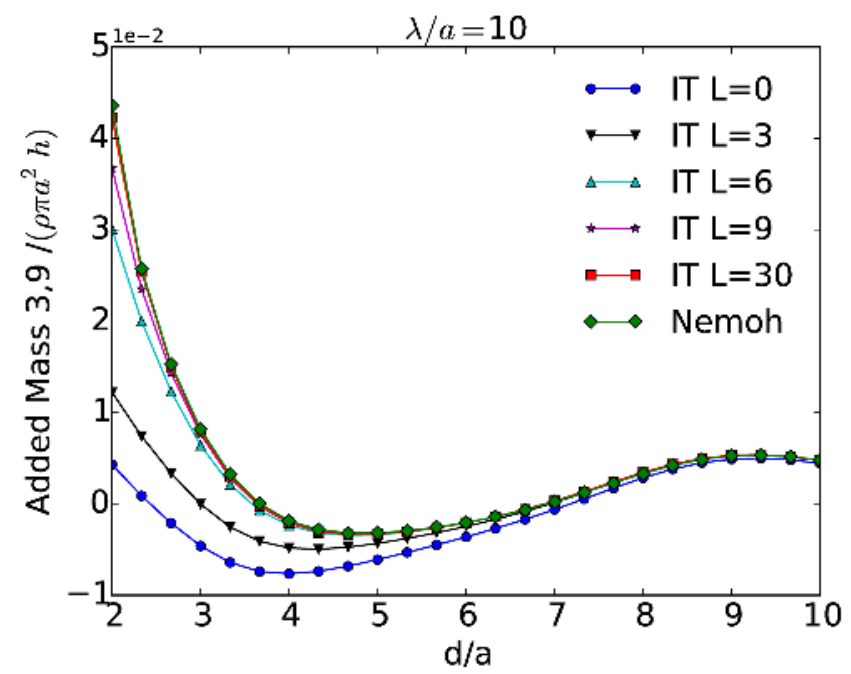

Figure 14: Heave-heave coupling added mass coefficient as a function of the evanescent modes truncation $L$ and for a small array of two truncated vertical circular cylinders of radius $a$, draft $h=2 a$ in water depth of $50 a / 3$.

As expected, the hydrodynamic coefficients are the same as if the cylinders were in isolation. Moreover, for such a long separating distance the evanescent modes do not play an important role. This can be derived from the fact that in their absence $(L=0)$ a very good agreement of results between the IT and the direct computations (Figure 13a) is obtained.

When bodies are set closer, in this case at a distance of $d / a=5$, hydrodynamic interactions become important as can be observed in Figures $13 p$ and 13 . For $k a>0.2$, the added mass and damping coefficients in isolation are altered. A similar behaviour was shown by Siddorn P. and Eatock Taylor (2008) for the heave coupling radiation hydrodyamic coefficients. At this separating distance $(d / a=5)$ and for the surge mode of motion, the influence of the evanescent modes is still negligible. This result is in agreement with Mavrakos, S. A. and 370 McIver (1997) who stated that the plane-wave approximation can provide accurate results when the separating distance between devices is larger than 5 times their characteristic dimension. 

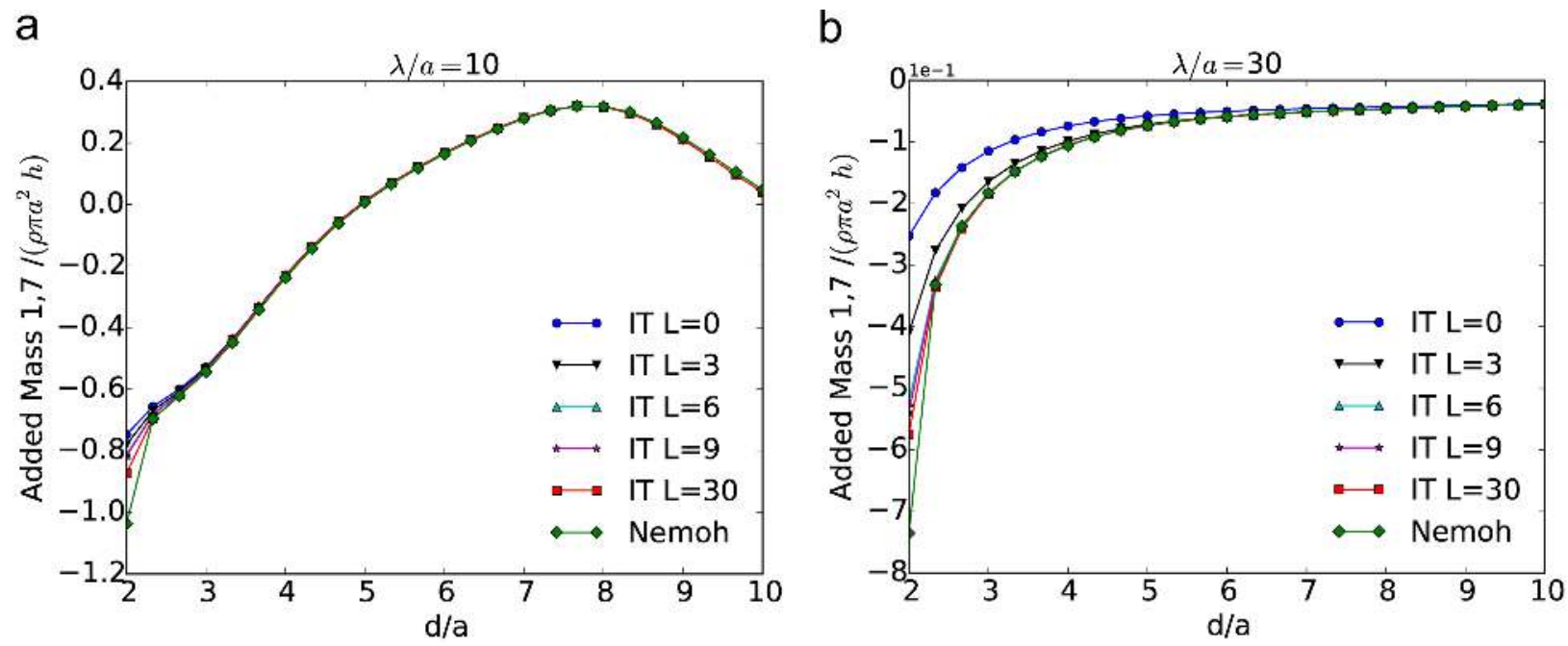

Figure 15: Surge-surge coupling added mass coefficient as a function of the evanescent modes truncation $L$ for two different wavelengths for a small array of two truncated vertical circular cylinders of radius $a$, draft $h=2 a$ in water depth of $50 a / 3$.

Finally, for a fixed frequency corresponding to a wave length of $\lambda / a=$ $10(k a=0.6)$, the coupling heave-heave added mass coefficient $(3,9)$ is shown (Figure 14) at several separating distances which span from $d / a=10$ to the limit case where the perimeters of both cylinders are externally tangent $(d / a=2)$. The influence of the evanescent modes for the heave-heave coupling can be clearly observed, as well as convergence of the IT results to direct calculation when the truncation of evanescent modes is increased. It has been observed that their importance to ensure accuracy of the radiation hydrodynamic coefficients (both added mass and damping) for close separating distances is mainly dependent on the motion mode and the frequency. For instance, for the surge-surge coupling, their influence at $\lambda / a=10$ (Figure $15 a$ ) is negligible. At a separation distance of $d / a=2.6$ the error is $3 \%$. This is not the case at $\lambda / a=30$ (Figure $15 p)$ where the error for the same separation distance is $40 \%$.

Another way to visualize the effect of the evanescent modes truncation consists of studying the free surface elevation $(\eta)$. Figures $17 a$ and $17 b$ show the total wave elevation, i.e. the sum of incident, scattered and radiated wave eleva- 


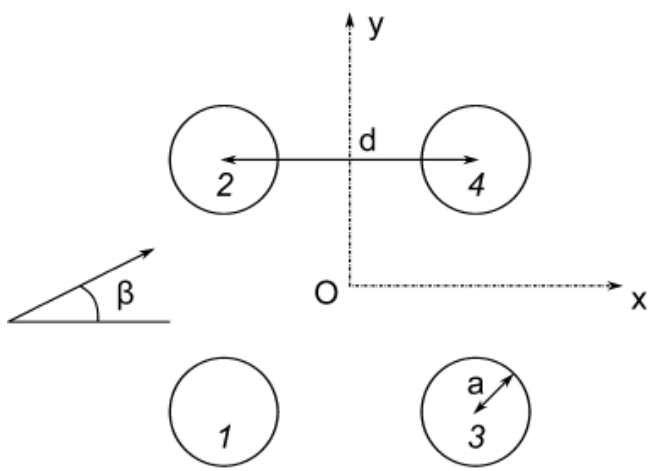

Figure 16: Schematic of an array composed of four truncated vertical cylinders of radius $a$ and separated by a distance between centers $d$. $\beta=0$ corresponds to the positive $x$-axis.

tions including the computed body motions, obtained with $\mathrm{NEMOH}$ and with the IT respectively for a small array of 4 freely floating truncated vertical cylinders for a regular wave of propagation direction $\beta=0$ and wavelength $\lambda / a=10$ with $a$ the radius of the cylinders. The IT free surface elevation has been calculated using its definition $\eta=-\left.\frac{1}{g} \frac{\partial \phi}{\partial t}\right|_{z=0}$, where the velocity potential in the fluid domain has been reconstructed from the solution of the multiple-scattering problem by means of expressions in (6).

It can be observed that a very good agreement between results is obtained for the whole domain (Figure 17f) when no evanescent modes are used with the highest differences being located at the vicinity of the bodies. The use of a higher evanescent modes truncation $17,17,17 f)$ reduces the error at these regions, from $4 \%$ to $0.4 \%$ at the mid-point of the line linking the centers of cylinders 2 and 4 (Figure 16), and results computed with the interaction theory converge with those obtained from direct calculations with the standard BEM code.

\subsection{Near Trapped Modes}

Siddorn P. and Eatock Taylor (2008) showed that the wave numbers prone to the physical phenomena of near-trapped modes, characterized by a large increase of the hydrodynamic force on one of the bodies of the array, can be detected 
a
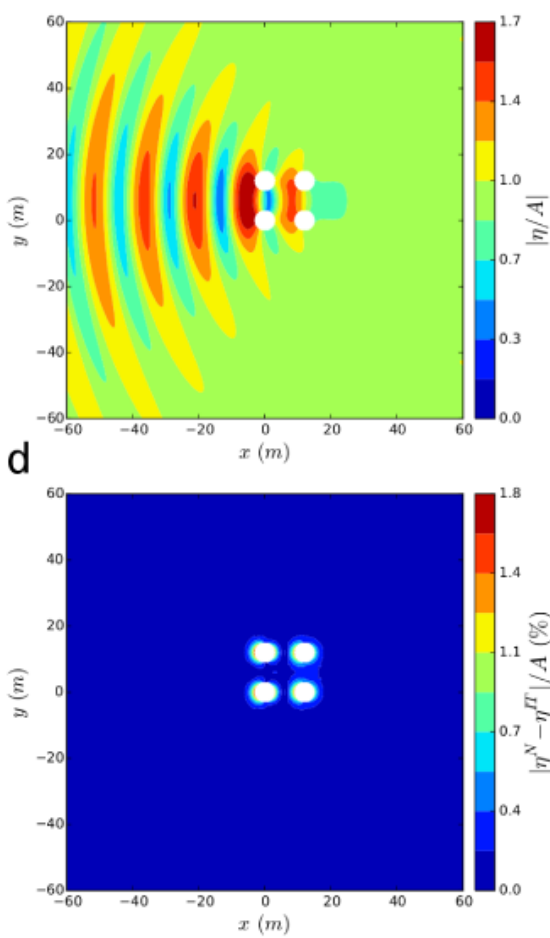

b

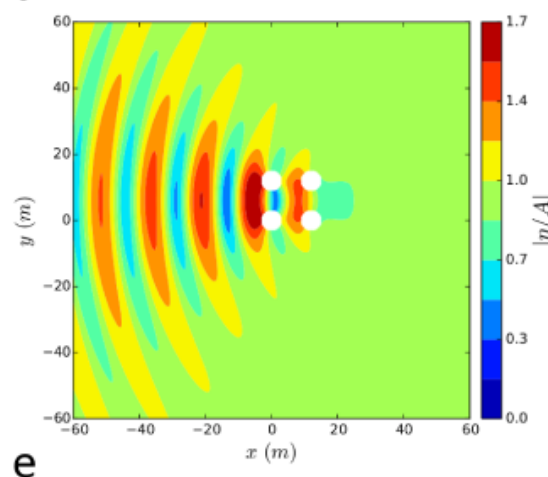

e

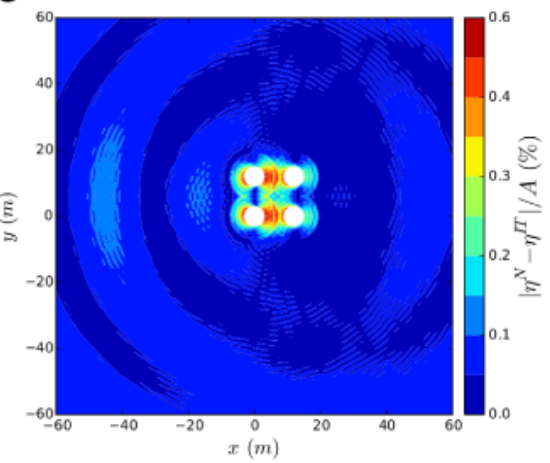

C

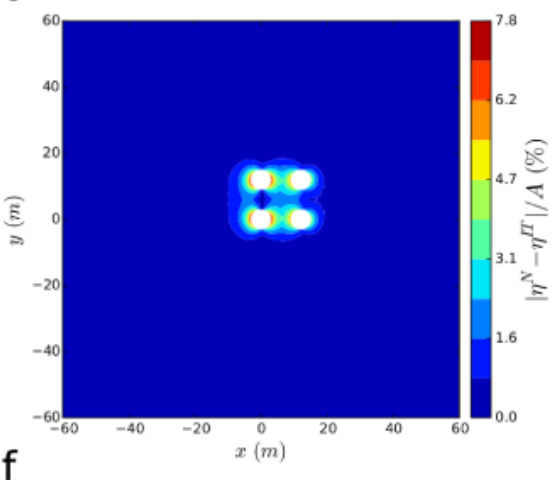

f

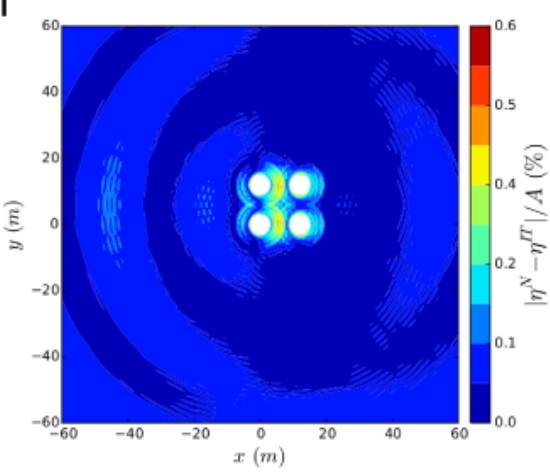

Figure 17: Magnitude of surface elevation for an array of 4 cylinders of radius $(a)$, draft $(2 a)$ in a water depth $(50 a / 3)$ with a separation distance of $4 a$. Subplot $a)$ shows the magnitude of the free surface elevation obtained from a direct NEMOH calculation and normalized by the amplitude of the incident wave, subplot $b$ ) shows the same result computed with the interaction theory (IT), and subplots $c), d), e), f$ ) show the percentage difference between the wave fields computed with the interaction theory and the direct NEMOH calculation as a function of the evanescent modes truncation $L((c)-L=0,(d)-L=6,(e)-L=12,(f)$ $L=18)$. Propagation direction is defined from left to right. 
through the peaks of the condition number of the array scattering matrix (19). An example of this is shown in Figure 18 for a small array of 4 truncated vertical cylinders of radius $a$, draft $2 a$ in a water depth of $4 a$ separated by a distance of $4 a$ (Figure 16). It can be observed that our results match the ones obtained by Siddorn P. and Eatock Taylor (2008), who used a semi-analytical solution instead of a BEM code to compute the hydrodynamic operators, except at $k a=2.44$ and $k a=3.85$ where small discrepancies occur due to the irregular frequencies. We note a slightly smaller condition number between $k a \sim 0.5$ and $k a \sim 1.5$. This may be due to the use of different depth truncation $(L=0$ for all $\mathrm{ka}$ ) and angular mode truncation values to the ones in Siddorn P. and Eatock Taylor (2008) which are not specified.

That the wave number matches one of the candidates identified from the analysis of the condition number does not imply that the near-trapped mode will occur. As mentioned by Siddorn P. and Eatock Taylor (2008) it is required as well that the wave heading is the correct one to excite it. For the four cylinder array under consideration, this happens when the wave heading is $\beta=\pi / 4$ and it produces very large excitation forces in surge at $k a \sim 1.66$ as shown in Figure ${ }_{425}$ 19. As in the previous case, the match between results is very good. A very high accuracy of the excitation force is obtained without making use of evanescent modes, which agrees with the observations of Chakrabarti (2000).

\section{Conclusions}

The calculation of the Diffraction Transfer Matrix and the Radiation Characteristics has been implemented in the open source BEM solver NEMOH using the methodology of Goo, J.-S. and Yoshida (1990). Results of the progressive terms of the hydrodynamic operators for a truncated vertical cylinder and a \$quare box have been contrasted with the methodology developed by McNatt J. C. et al. (2015) and a very good agreement has been found. In addition, the DTM and RC of the truncated vertical circular cylinder have been checked against the semi-analytical solution by Zeng and Tang (2013) and a very good 


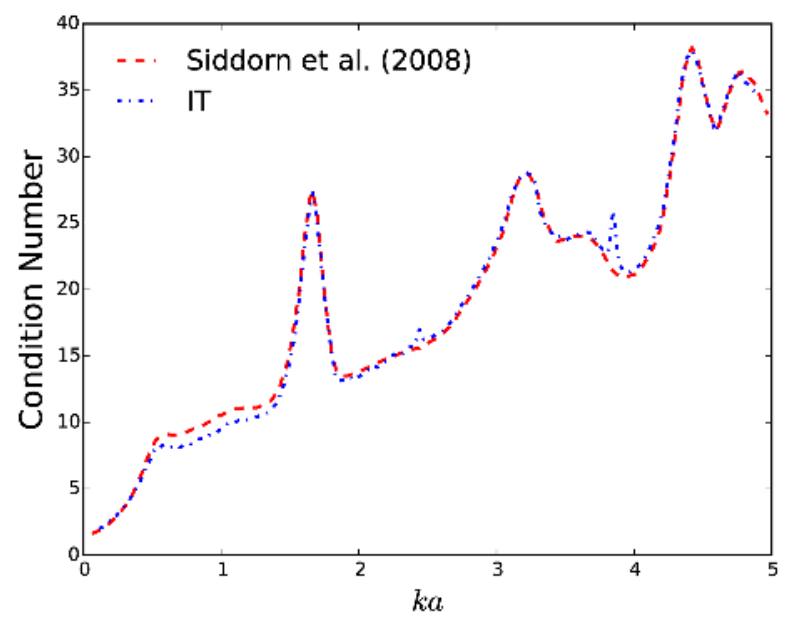

Figure 18: Variation of the condition number (computed using the 1-norm or maximum column-sum) of the scattering matrix of an array of 4 truncated vertical cylinders with radius $(a)$, draft $(h=2 a)$ in a water depth of $(4 a)$ with each cylinder disposed at the vertex of a square of side size $4 a$ (Figure 16 .

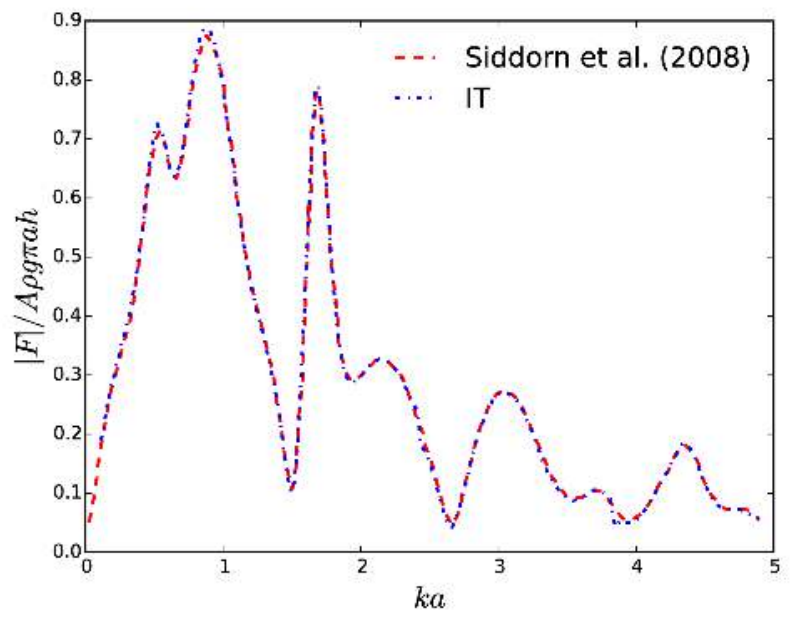

Figure 19: Variation of the surge excitation force on Cylinder 1 (Figure 16) of an array of 4 truncated vertical cylinders with radius $(a)$, draft $(h=2 a)$ in a water depth of $(4 a)$ with each cylinder disposed at the vertex of a square of side size $4 a$ for an incident wave heading angle of $\beta=\pi / 4$. The nondimensionalization factor terms are the wave amplitude $(A)$, the cylinder radius $(a)$ and the cylinder $\operatorname{draft}(h)$. 
match has been obtained.

For a truncated vertical cylinder, only the terms of the DTM corresponding to pairs of equal incident and outgoing angular modes have been observed to be different than zero. In contrast, for the square box, coupling terms between different incident and outgoing angular modes have been found to be significant. In both cases, the truncation of the angular terms has been shown to be frequency dependent. In addition, it has been checked that angular modes $m=-1,0,1$ are sufficient to represent the radiated waves of a truncated vertical circular cylinder in surge (odd terms) and heave (even term) for all frequencies. At the same time, it has been observed that higher modes are required, particularly at the high frequency zone, to represent the radiated wave in surge by a square box.

․ Radiation hydrodynamic coefficients computed with the IT by Kagemoto H. and Yue (1986) have been verified against direct BEM computations for a small array of two truncated vertical circular cylinders and a very good match of results has been observed. For closely spaced configurations, it is well known that the effect of hydrodynamic interactions and the evanescent modes truncation on the radiation coefficients is significant, and both are well-represented in our results. In addition, the free surface elevation of an array of four freely floating truncated vertical circular cylinders computed with the IT has been verified with direct BEM calculations. The effect of the evanescent modes truncation has been clearly shown to be significant only at the vicinity of the bodies as expected.

Finally, excitation forces computed with the IT for a 4 cylinder array configuration prone to trapped-modes have been verified against semi-analytical results by Siddorn P. and Eatock Taylor (2008) and a very good agreement has been found. In contrast to the radiation coefficients, it has been observed that in this case the influence of the evanescent modes truncation is negligible.

465 The numerical tool that we have described and verified in this study can be used for the frequency domain simulation of large arrays of floating bodies, such as wave energy converters. The gain in computational speed when compared to 
direct calculations using standard BEM codes makes it suitable for optimization purposes.

\section{Acknowledgements}

The research leading to these results is part of the OceaNET project, which has received funding from the European Union's Seventh Framework Programme for research, technological development and demonstration under grant agreement no 607656 .

\section{References}

Abramowitz M. , Segun A. I. Handbook of mathematical functions with Formulas, Graphs, and Mathematical Tables. 1st ed. Dover Publications, Inc, 1964.

Babarit A. , Delhommeau G. Theoretical and numerical aspects of the open source BEM solver NEMOH. In: Proceedings of the 11th European Wave and Tidal Energy Conference 6-11th Sept 2015, Nantes, France. 2015. p. 1-12.

Budal K. Theory for Absorption of Wave Power by a System of Interacting Bodies. Journal of Ship Research 1977;21(4):248-53.

Chakrabarti S. Hydrodynamic interaction forces on multi-moduled structures. Ocean Engineering 2000;27(10):1037-63.

Chakrabarti S. Response due to moored multiple structure interaction. Marine Structures 2001;14(1-2):231-58.

Child B. On the configuration of arrays of floating wave energy converters. Ph.D. thesis; The University of Edinburgh; 2011. 
Crooks D, Hoff JV, Folley M, Elsaesser B. Oscillating Wave Surge Converter Forced Oscillation Tests. In: Proceedings of the ASME 2016 35th International Conference on Ocean, Offshore and Arctic Engineering. 2016. p. 1-10.

Falnes J. Wave-power absorption by an array of attenuators oscillating with unconstrained amplitudes. Applied Ocean Research 1984;6(1):16 - 22.

Falnes J., Budal K. Wave-power absorption by parallel rows of interacting oscillating bodies. Applied Ocean Research 1982;4:194 - 207.

Fenton J. Wave forces on vertical bodies of revolution. Journal of Fluid Mechanics 1978;85:241-55.

Goo, J.-S. , Yoshida K. A Numerical Method for Huge Semisubmersible Responses in Waves. Society of Naval Architects and Marine Engineers 1990;98:365-87.

Göteman, M. , Engström, J. , Eriksson, M. , Isberg J. Fast Modeling of Large Wave Energy Farms Using Interaction Distance Cut-Off. Energies 2015;8(12):13741-57.

Kagemoto H. , Yue D. Interactions among multiple three-dimensional bodies in water waves: an exact algebraic method. Journal of Fluid Mechanics 1986;166(-1):189.

Kashiwagi M. Hydrodynamic interactions among a great number of columns supporting a very large flexible structure. Journal of Fluids and Structures 2000;14:1013-34.

Kashiwagi M. Wave scattering among a large number of floating cylinders. Journal of Structural Engineering and Mechanics 2005;21:53-66.

Kashiwagi M. 3-D Calculation for Multiple Floating Bodies in Proximity Using Wave Interaction Theory. International Journal of Offshore and Polar Engineering 2008;18(1):14-20. 
Martin P. A. Multiple Scattering: Interaction of Time-Harmonic Waves with N Obstacles. 2006.

Mavrakos, S. A. , McIver P. Comparison of methods for computing hydrodynamic characteristics of arrays of wave power devices. Applied Ocean Research 1997;1187(97):283-91.

McNatt J. C. , Venugopal, V. , Forehand D. The cylindrical wave field of wave energy converters. International Journal of Marine Energy 2013;3-4:e26-39.

McNatt J. C. , Venugopal, V. , Forehand D. A novel method for deriving the diffraction transfer matrix and its application to multi-body interactions in water waves. Ocean Engineering 2015;94:173-85.

Montiel F., Squire V. A. , Bennetts L. Attenuation and directional spreading of ocean wave spectra in the marginal ice zone. Journal of Fluid Mechanics $530 \quad 2016 ; 790: 492-522$.

Newman, J.N . Marine Hydrodynamics. 9th ed. The Massachusetts Institute of Technology, 1999.

Ohkusu M. Hydrodynamic forces on multiple cylinders in waves. In: International Symposium on the Dynamics of Marine Vehicles and Structures in Waves. 1974. p. 107-12.

Peter M. A. , Meylan H. M. Infinite-depth interaction theory for arbitrary floating bodies applied to wave forcing of ice floes. Journal of Fluid Mechanics 2004;500:145-67.

Siddorn P. , Eatock Taylor R. Diffraction and independent radiation by an array of floating cylinders. Ocean Engineering 2008;35(13):1289-303.

Simon M. Multiple scattering in arrays of axisymmetric wave-energy devices. Part 1. A matrix method using a plane-wave approximation. Journal of Fluid Mechanics 1982;120(-1):1. 
Spring B. H. , Monkmeyer P. L. Interaction of Plane Waves With Vertical Cylinders. Proceedings of the 14th Coastal Engineering Proceedings 1974;3:182847.

Twersky V. Multiple scattering and radiation by an arbitrary configuration of parallel cylinders. Journal of the Acoustical Society of America 1952;24:42-6.

Watson G. A treatise on the theory of Bessel functions. Cambridge University Press, 1966.

Zeng Xh, Tang Sy. The hydrodynamic interactions of an array of truncated circular cylinders as each cylinder oscillates independently with different prescribed modes. Journal of Hydrodynamics, Ser B 2013;25(1):27-38. 